\title{
Matching Hom-Setting of Rota-Baxter Algebras, Dendriform Algebras, and Pre-Lie Algebras
}

\author{
Dan Chen, ${ }^{1}$ Xiao-Song Peng, ${ }^{1}$ Chia Zargeh, ${ }^{2}$ and Yi Zhang $\mathbb{D}^{1,3}$ \\ ${ }^{1}$ School of Mathematics and Statistics, Lanzhou University, Lanzhou, Gansu 730000, China \\ ${ }^{2}$ Instituto de Matemática e Estatística, Universidade de São Paulo, São Paulo, SP, Brazil \\ ${ }^{3}$ School of Mathematics and Statistics, NUIST, Nanjing, Jiangsu 210044, China
}

Correspondence should be addressed to Yi Zhang; zhangy2016@lzu.edu.cn

Received 11 February 2020; Revised 10 May 2020; Accepted 1 June 2020; Published 10 July 2020

Academic Editor: Yao-Zhong Zhang

Copyright (C) 2020 Dan Chen et al. This is an open access article distributed under the Creative Commons Attribution License, which permits unrestricted use, distribution, and reproduction in any medium, provided the original work is properly cited.

In this paper, we introduce the Hom-algebra setting of the notions of matching Rota-Baxter algebras, matching (tri)dendriform algebras, and matching pre-Lie algebras. Moreover, we study the properties and relationships between categories of these matching Hom-algebraic structures.

\section{Introduction}

1.1. Hom-Algebraic Structures. The origin of Hom-structures may be found in the study of Hom-Lie algebras which were first introduced by Hartwig, Larsson, and Silvestrov [1]. Hom-Lie algebras, as a generalization of Lie algebras, are introduced to describe the structures on deformations of the Witt algebra and the Virasoro algebra. More precisely, a Hom-Lie algebra is a triple $(L,[-,-], \alpha)$ consisting of a $\mathrm{k}-$ module $L$, a bilinear skew-symmetric bracket $[-,-]: L \otimes$ $L \longrightarrow L$ and an algebra endomorphism $\alpha: L \longrightarrow L$ satisfying the following Hom-Jacobi identity:

$$
\begin{aligned}
& {[\alpha(x),[y, z]]+[\alpha(y),[z, x]]+[\alpha(x),[x, y]]} \\
& \quad=0 \text { for all } x, y, z \in L .
\end{aligned}
$$

Recently, there have been several interesting developments of Hom-Lie algebras in mathematics and mathematical physics, including Hom-Lie bialgebras [2, 3], quadratic Hom-Lie algebras [4], involutive Hom-semigroups [5], deformed vector fields and differential calculus [6], representations $[7,8]$, cohomology and homology theory $[9,10]$, Yetter-Drinfeld categories [11], Hom-Yang-Baxter equations [12-16], Hom-Lie 2-algebras [17, 18], $(m, n)$-Hom-Lie alge- bras [19], Hom-left-symmetric algebras [20], and enveloping algebras [21]. In particular, the Hom-Lie algebra on semisimple Lie algebras was studied in [22], and the Hom-Lie structure on affine Kac-Moody was constructed in [23].

In 2008, Makhlouf and Silvestrov [20] introduced the notation of Hom-associative algebras whose associativity law is twisted by a linear map. Usual functors between the categories of Lie algebras and associative algebras have been extended to the Hom-setting. It is shown that a Homassociative algebra gives rise to a Hom-Lie algebra using the commutator. Since then, various Hom-analogues of some classical algebraic structures have been introduced and studied intensively, such as Hom-coalgebras, Hom-bialgebras and Hom-Hopf algebras [24, 25], Hom-groups [26, 27], Hom-Hopf modules [28], Hom-Lie superalgebras [29, 30], generalize Hom-Lie algebras [31], and Hom-Poisson algebras [32].

Dendriform algebras were introduced by Loday [33] with motivation from algebraic $K$-theory. Latter, tridendriform algebras were proposed by Loday and Ronco [34] in their study of polytopes and Koszul duality. The classical links between Rota-Baxter algebras and (tri)dendriform algebras were given in $[35,36]$, resembling the structure of Lie algebras on an associative algebra. In 2012, Makhlouf [37] generalized the concepts of dendriform algebras and Rota-Baxter 


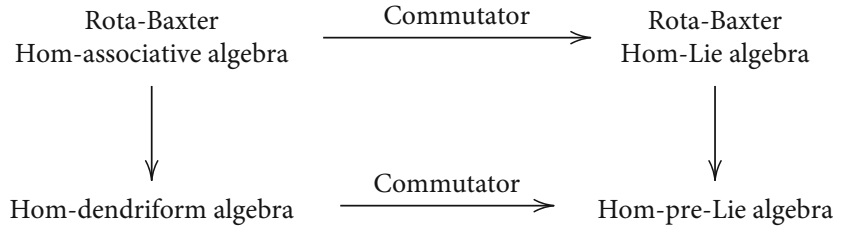

FIGURE 1

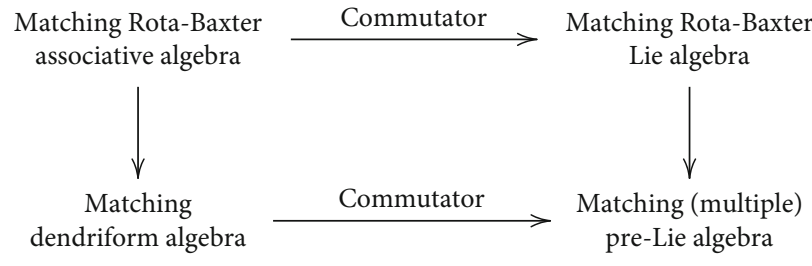

FIGURE 2 algebras by twisting the identities by mean of a linear map, which were called Hom-dendriform algebras and RotaBaxter Hom-algebras, respectively. The connections between all these categories of Hom algebras were also investigated in [37]. Due to the fundamental work of Makhlouf [37], we have the following commutative diagram of categories (the arrows will go in the opposite direction for the corresponding operads), see Figure 1.

1.2. Motivations for Matching Hom-Algebraic Structures. The recent concept of a matching or multiple Rota-Baxter [38] came from the study of multiple pre-Lie algebras [39] originated from the pioneering work of Bruned, Hairer, and Zambotti [40] on algebraic renormalization of regularity structures. It is shown that the matching Rota-Baxter algebra was motivated by the studies of associative Yang-Baxter equations, Volterra integral equations, and linear structure of Rota-Baxter operators [38]. More precisely, for exploring the relationship between associative Yang-Baxter equations and classical Yang-Baxter equations, Aguiar [41] proposed a polarized form of the expression on the left-hand side of the associative Yang-Baxter equation:

$$
\{r, s\}:=r_{13} s_{12}-r_{12} s_{23}+r_{23} s_{13}
$$

where $r, s \in A \otimes A$ and $A$ is a unitary associative algebra. The corresponding equation

$$
r_{13} s_{12}-r_{12} s_{23}+r_{23} s_{13}=0
$$

was called polarized associative Yang-Baxter equation (PAYBE) by Guo and etc. [38]. Paralleled to the fact that solutions of the associative Yang-Baxter equation naturally give Rota-Baxter operators, the matching Rota-Baxter operators are determined by solutions of a PAYBE [38].

The basic theory of matching Rota-Baxter algebras was originally established in $[38,42]$, has proven useful not only in (compatible) multiple operations [43-48] but also in other areas of mathematics as well, such as polarized associative Yang-Baxter equation [38], algebraic combinatorics [38, 49], matching shuffle product [42], algebraic integral equation [50], and Gröbner-Shirshov bases and Hopf algebras [49]. Based on the close relationships between matching Rota-Baxter algebras, matching dendriform algebras, and matching pre-Lie algebras, Guo et al. [38] previously showed the following commutative diagram of categories, see Figure 2.

The main purpose of this paper is to extend these matching algebraic structures to the Hom-algebra setting and study the connections between these categories of Hom-algebras. These results give rise to the following commutative diagram of categories, see Figure 3.

We would like to emphasize that the notation of matching Hom-Lie Rota-Baxter algebras will play a curial role in mathematical physics. The Rota-Baxter equation on a Lie algebra is the operator form of the classical Yang-Baxter equation [51]. Similarly, there should be a close relationship between the matching Hom Rota-Baxter equation in (82) with weight zero and the polarized classical Yang-Baxter equation, as a Hom-Lie algebra variation of the Hom version of the polarized associative Yang-Baxter equation.

1.3. Outline of the Paper and Summary of Results. In section 2 , we provide definitions concerning the generalization of matching associative algebras, matching pre-Lie algebras to Hom-algebras setting and describe some specific cases of matching Hom-algebraic structures. Also, the close relationship between matching Hom-Lie algebras and Hom-Lie algebras will be shown.

In section 3, we extend the notion of matching RotaBaxter algebras to the Hom-associative algebra setting. It is also shown that matching Hom-associative Rota-Baxter algebras can be reduced from a matching Rota-Baxter algebra. At the end of this section, the construction of Hom-algebras using elements of the centroid is generalized to the matching Rota-Baxter algebras.

Section 4 is devoted to the definition of matching Hom(tri)dendriform algebras and the approach of construction of a matching Hom-(tri)dendiform algebra from a matching (tri)dendiform algebra. Some results related to the connections between matching Hom-(tri)dendiform algebras and compatible Hom-associative algebras as well as between matching Hom-dendriform algebras and matching HompreLie algebras will be established.

In section 5, the concepts of matching Hom-Lie RotaBaxter algebras and matching Rota-Baxter algebras involving elements of the centroid of matching Lie Rota-Baxter algebras will be established. Also, some results related to the connection between matching Hom-Lie Rota-Baxter algebra of weight zero and matching Hom-preLie algebra will be obtained.

Notation. Throughout this paper, let $k$ be a unitary commutative ring unless the contrary is specified, which will be the base ring of all modules, algebras, tensor products, operations as well as linear maps. We always suppose that $\Omega$ is a nonempty set. We denote by $P_{\Omega}:=\left(P_{\omega}\right)_{\omega \in \Omega}$ the collection of operations $P_{\omega}, \omega \in \Omega$, where $\Omega$ is a set indexing the linear operators. 


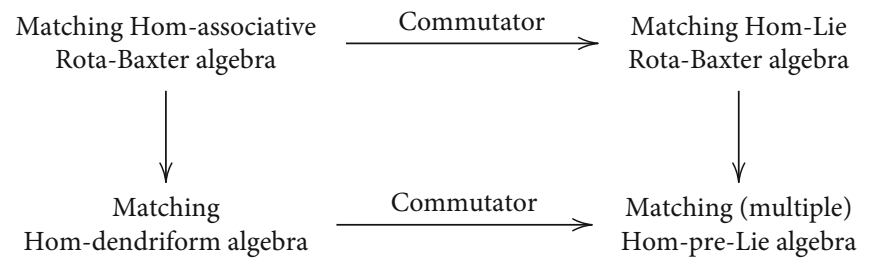

Figure 3

\section{Matching Hom-Associative, Matching Hom- preLie and Matching Hom-Lie Algebras}

In this section, we give the definitions of matching Homassociative algebras, compatible Hom-associative algebras, compatible Hom-preLie algebras, and compatible Hom-Lie algebras, which generalize the corresponding matching algebraic structures introduced in [38]. Then, we explore the relationships between these categories from the point of view of Hom-algebras.

Definition 1. A matching Hom-associative algebra is a kmodule $A$ together with a collection of binary operations ${ }_{\omega}: A \otimes A \longrightarrow A, \omega \in \Omega$ and a linear map $p: A \longrightarrow A$ such that

$$
\left(x \cdot{ }_{\alpha} y\right) \cdot{ }_{\beta} p(z)=p(x) \cdot{ }_{\alpha}\left(y \cdot{ }_{\beta} z\right) \text { for all } x, y, z \in A \text { and } \alpha, \beta \in \Omega \text {. }
$$

A matching Hom-associative algebra is called totally compatible if it satisfies

$$
\left(x \cdot{ }_{\alpha} y\right) \cdot{ }_{\beta} p(z)=p(x) \cdot{ }_{\beta}\left(y \cdot{ }_{\alpha} z\right) \text { for all } x, y, z \in A \text { and } \alpha, \beta \in \Omega \text {. }
$$

More generally,

Definition 2. A compatible Hom-associative algebra is a $\mathrm{k}$ module $A$ together with a collection of binary operations ${ }_{\omega}: A \otimes A \longrightarrow A, \omega \in \Omega$ and a linear map $p: A \longrightarrow A$ such that

$$
\left(x \cdot{ }_{\alpha} y\right) \cdot{ }_{\beta} p(z)+\left(x \cdot{ }_{\beta} y\right) \cdot{ }_{\alpha} p(z)=p(x) \cdot{ }_{\alpha}\left(y \cdot{ }_{\beta} z\right)+p(x) \cdot{ }_{\beta}\left(y \cdot{ }_{\alpha} z\right)
$$

for all $x, y, z \in A$ and $\alpha, \beta \in \Omega$. For simplicity, we denote it by $\left(A, \cdot_{\Omega}, p\right)$.

Remark 3.

(a) Any matching Hom-associative algebra or totally compatible Hom-associative algebra is a compatible Hom-associative algebra

(b) By taking $p=\mathrm{i} d$, we recover to the definition of matching associative algebras, totally compatible associative algebra and compatible associative algebra given in [38] (c) If $\Omega$ is a singleton and the characteristic of $k$ is not 2, then the notation of matching Hom-associative algebras and the notation of compatible Hom-associative algebras are equivalent and recover to the Homassociative algebras introduced in [20]

Definition 4. A matching Hom-Lie algebra is a k-module $\mathfrak{g}$ equipped with a collection of binary operations $[,]_{\omega}: \mathfrak{g} \otimes$ $\mathfrak{g} \longrightarrow \mathfrak{g}, \omega \in \Omega$ and a linear map $p: \mathfrak{g} \longrightarrow \mathfrak{g}$ such that

$$
\begin{aligned}
{[x, x]_{\omega} } & =0 \\
{\left[p(x),[y, z]_{\beta}\right]_{\alpha}+\left[p(y),[z, x]_{\alpha}\right]_{\beta}+\left[p(z),[x, y]_{\alpha}\right]_{\beta} } & =0
\end{aligned}
$$

for all $x, y, z \in \mathfrak{g}$ and $\alpha, \beta, \omega \in \Omega$.

Remark 5. A totally compatible Hom-associative algebra $(A, \cdot \Omega, p)$ has a natural matching Hom-Lie algebra structure with the Lie bracket defined by

$$
[x, y]_{\omega}:=x \cdot{ }_{\omega} y-y \cdot{ }_{\omega} x \text {, for } x, y \in A \text { and } \omega \in \Omega \text {. }
$$

The matching Hom-Lie algebra has a close relationship with Hom-Lie algebras. We first record a lemma for a preparation.

Lemma 6. Let $\left(\mathfrak{g},[,]_{\Omega}, p\right)$ be a matching Hom-Lie algebra. Consider linear combinations

$$
[,]_{A}:=\sum_{\alpha \in \Omega} a_{\alpha}[,]_{\alpha} \text { and }[,]_{B}:=\sum_{\beta \in \Omega} b_{\beta}[,]_{\beta} \text {, }
$$

where $a_{\alpha}, b_{\beta} \in k$ for $\alpha, \beta \in \Omega$ with finite supports. Then

$$
\begin{aligned}
& {\left[p(x),[y, z]_{B}\right]_{A}+\left[p(y),[z, x]_{A}\right]_{B}+\left[p(z),[x, y]_{A}\right]_{B}} \\
& \quad=0 \text { for } x, y, z \in \mathfrak{g} .
\end{aligned}
$$

Proof. By Eq. (10), for $x, y, z \in \mathfrak{g}$, we have

$$
\begin{aligned}
{\left[p(x),[y, z]_{B}\right]_{A} } & =\left[p(x), \sum_{\beta \in \Omega} b_{\beta}[y, z]_{\beta}\right]_{A} \\
& =\sum_{\alpha \in \Omega} a_{\alpha}\left[p(x), \sum_{\beta \in \Omega} b_{\beta}[y, z]_{\beta}\right]_{\alpha} \\
& =\sum_{\alpha \in \Omega} \sum_{\beta \in \Omega} a_{\alpha} b_{\beta}\left[p(x),[y, z]_{\beta}\right]_{\alpha} .
\end{aligned}
$$


Similarly, we also have

$$
\begin{aligned}
& {\left[p(y),[z, x]_{A}\right]_{B}=\sum_{\alpha \in \Omega} \sum_{\beta \in \Omega} b_{\beta} a_{\alpha}\left[p(y),[z, x]_{\alpha}\right]_{\beta} \text { and }} \\
& {\left[p(z),[x, y]_{A}\right]_{B}=\sum_{\alpha \in \Omega} \sum_{\beta \in \Omega} b_{\beta} a_{\alpha}\left[p(z),[x, y]_{\alpha}\right]_{\beta} .}
\end{aligned}
$$

Since $\left(\mathfrak{g},[,]_{\Omega}, p\right)$ is a matching Hom-Lie algebra, then

$$
\begin{aligned}
& {\left[p(x),[y, z]_{\beta}\right]_{\alpha}+\left[p(y),[z, x]_{\alpha}\right]_{\beta}+\left[p(z),[x, y]_{\alpha}\right]_{\beta}} \\
& \quad=0 \text { for all } x, y, z \in \mathfrak{g} \text { and } \alpha, \beta \in \Omega
\end{aligned}
$$

Thus

$$
\left[p(x),[y, z]_{B}\right]_{A}+\left[p(y),[z, x]_{A}\right]_{B}+\left[p(z),[x, y]_{A}\right]_{B}=0
$$

as desired.

Proposition 7. Let $\left(\mathfrak{g},[,]_{\Omega}, p\right)$ be a matching Hom-Lie algebra. Consider linear combinations

$$
[,]_{A}:=\sum_{\omega \in \Omega} a_{\omega}[]_{\omega}, a_{\omega} \in k
$$

with a finite support. Then, $\left(\mathfrak{g},[,]_{A}\right)$ is a Hom-Lie algebra.

Proof. It follows from Lemma 6 by taking $\left(a_{\omega}\right)_{\omega \in \Omega}=\left(b_{\omega}\right)_{\omega \in \Omega}$.

More generally, we propose

Definition 8. A compatible Hom-Lie algebra is a k-module $\mathfrak{g}$ together with a set of binary operations [,] $: \mathfrak{g} \otimes \mathfrak{g} \longrightarrow \mathfrak{g}$, $\omega \in \Omega$ and a linear map $p: \mathfrak{g} \longrightarrow \mathfrak{g}$ such that

$$
\begin{gathered}
{[x, x]_{\omega}=0} \\
{\left[p(x),[y, z]_{\alpha}\right]_{\beta}+\left[p(y),[z, x]_{\alpha}\right]_{\beta}+\left[p(z),[x, y]_{\alpha}\right]_{\beta}} \\
+\left[p(x),[y, z]_{\beta}\right]_{\alpha}+\left[p(y),[z, x]_{\beta}\right]_{\alpha}+\left[p(z),[x, y]_{\beta}\right]_{\alpha}=0
\end{gathered}
$$

for all $x, y, z \in \mathfrak{g}$ and $\omega, \alpha, \beta \in \Omega$.

\section{Remark 9.}

(a) Every matching Hom-Lie algebra is a compatible Hom-Lie algebra.

(b) Given two Hom-Lie algebras $\left(\mathfrak{g},[,]_{\alpha}, p\right)$ and $\left(\mathfrak{g},[,]_{\beta}, p\right)$. Define a new bracket [,]: $\mathfrak{g} \otimes \mathfrak{g} \longrightarrow \mathfrak{g}$ as follows:

$$
[x, y]:=a_{\alpha}[x, y]_{\alpha}+b_{\beta}[x, y]_{\beta} \text { for some } a_{\alpha}, b_{\beta} \in k
$$

Clearly, this new bracket is both skew symmetric and bilinear. Then, $(\mathfrak{g},[], p$,$) is further a Hom-Lie algebra if [,] sat-$ isfies the Hom-Jacobi identity

$$
[p(x),[y, z]]+[p(y),[z, x]]+[p(z),[x, y]]=0
$$

By a direct calculation, we get that this condition is equivalent to Eq. (18).

Proposition 10. Let $\left(\mathfrak{g},[,]_{\Omega}, p\right)$ be a matching Hom-Lie algebra. Then for $x, y, z \in \mathfrak{g}$ and $\alpha, \beta \in \Omega$, we have

$$
\begin{aligned}
& {\left[p(x),[y, z]_{\alpha}\right]_{\beta}=\left[p(x),[y, z]_{\beta}\right]_{\alpha},} \\
& {\left[p(x),[y, z]_{\alpha}\right]_{\beta}+\left[p(y),[z, x]_{\alpha}\right]_{\beta}+\left[p(z),[x, y]_{\alpha}\right]_{\beta}=0 .}
\end{aligned}
$$

Proof. Since Eq. (8) holds for any $x, y, z \in A$ and $\alpha, \beta \in \Omega$, we get

$$
\left[p(y),[z, x]_{\alpha}\right]_{\beta}+\left[p(z),[x, y]_{\beta}\right]_{\alpha}+\left[p(x),[y, z]_{\beta}\right]_{\alpha}=0 .
$$

Eqs. (8) and (22) result in

$$
\left[p(z),[x, y]_{\alpha}\right]_{\beta}-\left[p(z),[x, y]_{\beta}\right]_{\alpha}=0
$$

By the arbitrariness of $x, y, z$, we have

$$
\left[p(x),[y, z]_{\alpha}\right]_{\beta}=\left[p(x),[y, z]_{\beta}\right]_{\alpha}
$$

and so

$$
\left[p(x),[y, z]_{\alpha}\right]_{\beta}+\left[p(y),[z, x]_{\alpha}\right]_{\beta}+\left[p(z),[x, y]_{\alpha}\right]_{\beta}=0 .
$$

Generalizing the well-known result that an associative algebra has a Lie algebra structure via the commutator bracket, we show that a compatible Hom-associative algebra has a compatible Hom-Lie algebra structure.

Proposition 11. Let $\left(A,{ }_{\Omega}, p\right)$ be a compatible Homassociative algebra. Then $\left(A,[,]_{\Omega}, p\right)$ is a compatible HomLie algebra, where

[]$_{\omega}: A \otimes A \longrightarrow A,[x, y]_{\omega}:=x \cdot{ }_{\omega} y-y \cdot{ }_{\omega} x$ for $x, y \in A$ and $\omega \in \Omega$.

Proof. For $x, y, z \in A$ and $\alpha, \beta, \omega \in \Omega$, by Eq. (26), we get $[x, x]_{\omega}=0$ and

$$
\begin{aligned}
{\left[p(x),[y, z]_{\alpha}\right]_{\beta}=} & {\left[p(x), y \cdot{ }_{\alpha} z-z \cdot{ }_{\alpha} y\right]_{\beta} } \\
= & p(x) \cdot{ }_{\beta}\left(y \cdot{ }_{\alpha} z-z \cdot{ }_{\alpha} y\right)-\left(y \cdot{ }_{\alpha} z-z \cdot{ }_{\alpha} y\right) \cdot{ }_{\beta} p(x) \\
= & p(x) \cdot{ }_{\beta}\left(y \cdot{ }_{\alpha} z\right)-p(x) \cdot{ }_{\beta}\left(z \cdot{ }_{\alpha} y\right) \\
& -\left(y \cdot{ }_{\alpha} z\right) \cdot{ }_{\beta} p(x)+\left(z \cdot{ }_{\alpha} y\right) \cdot{ }_{\beta} p(x) .
\end{aligned}
$$


Similarly, we have

$$
\begin{aligned}
{\left[p(y),[z, x]_{\alpha}\right]_{\beta}=} & p(y) \cdot{ }_{\beta}\left(z \cdot{ }_{\alpha} x\right)-p(y) \cdot{ }_{\beta}\left(x \cdot{ }_{\alpha} z\right) \\
& -\left(z \cdot \cdot_{\alpha} x\right) \cdot{ }_{\beta} p(y)+\left(x \cdot{ }_{\alpha} z\right) \cdot{ }_{\beta} p(y), \\
{\left[p(z),[x, y]_{\alpha}\right]_{\beta}=} & p(z) \cdot{ }_{\beta}\left(x \cdot{ }_{\alpha} y\right)-p(z) \cdot \cdot_{\beta}\left(y \cdot{ }_{\alpha} x\right) \\
& -\left(x \cdot{ }_{\alpha} y\right) \cdot{ }_{\beta} p(z)+\left(y \cdot{ }_{\alpha} x\right) \cdot{ }_{\beta} p(z), \\
{\left[p(x),[y, z]_{\beta}\right]_{\alpha}=} & p(x) \cdot{ }_{\alpha}\left(y \cdot{ }_{\beta} z\right)-p(x) \cdot{ }_{\alpha}\left(z \cdot{ }_{\beta} y\right) \\
& -\left(y \cdot{ }_{\beta} z\right) \cdot{ }_{\alpha} p(x)+\left(z \cdot{ }_{\beta} y\right) \cdot{ }_{\alpha} p(x), \\
{\left[p(y),[z, x]_{\beta}\right]_{\alpha}=} & p(y) \cdot{ }_{\alpha}\left(z \cdot{ }_{\beta} x\right)-p(y) \cdot{ }_{\alpha}\left(x \cdot \cdot_{\beta} z\right) \\
& -\left(z \cdot{ }_{\beta} x\right) \cdot{ }_{\alpha} p(y)+\left(x \cdot{ }_{\beta} z\right) \cdot{ }_{\alpha} p(y), \\
{\left[p(z),[z, y]_{\beta}\right]_{\alpha}=} & p(z) \cdot{ }_{\alpha}\left(x \cdot{ }_{\beta} y\right)-p(z) \cdot{ }_{\alpha}\left(y \cdot{ }_{\beta} x\right) \\
& -\left(x \cdot{ }_{\beta} y\right) \cdot{ }_{\alpha} p(z)+\left(y \cdot{ }_{\beta} x\right) \cdot{ }_{\alpha} p(z) .
\end{aligned}
$$

By Eq. (6), we get

$$
\begin{gathered}
{\left[p(x),[y, z]_{\alpha}\right]_{\beta}+\left[p(y),[z, x]_{\alpha}\right]_{\beta}+\left[p(z),[x, y]_{\alpha}\right]_{\beta}} \\
+\left[p(x),[y, z]_{\beta}\right]_{\alpha}+\left[p(y),[z, x]_{\beta}\right]_{\alpha}+\left[p(z),[x, y]_{\beta}\right]_{\alpha}=0 .
\end{gathered}
$$

Hence, $\left(A,[,]_{\Omega}, p\right)$ is a compatible Hom-Lie algebra.

Now, we give the definition of matching Hom-preLie algebras.

Definition 12. A matching Hom-preLie algebra is a k-module $A$ together with a family of binary operations $*_{\omega}: A \otimes A$ $\longrightarrow A, \omega \in \Omega$ and a linear map $p: A \longrightarrow A$ such that

$$
\begin{aligned}
& p(x) *_{\alpha}\left(y *_{\beta} z\right)-\left(x *_{\alpha} y\right) *_{\beta} p(z) \\
& \quad=p(y) *_{\beta}\left(x *_{\alpha} z\right)-\left(y *_{\beta} x\right){ }_{\alpha} p(z)
\end{aligned}
$$

for all $x, y, z \in A$ and $\alpha, \beta \in \Omega$.

Now, we give the relationship between matching HompreLie algebras and compatible Hom-Lie algebras.

Proposition 13. Let $\left(A, *_{\Omega}, p\right)$ be a matching Hom-preLie algebra. Then $\left(A,[,]_{\Omega}, p\right)$ is a compatible Hom-Lie algebra, where

$$
\begin{aligned}
{[]_{\omega} } & : A \otimes A \longrightarrow A,[x, y]_{\omega} \\
& :=x *{ }_{\omega} y-y *{ }_{\omega} x, \text { for all } x, y \in A \text { and } \omega \in \Omega .
\end{aligned}
$$

Proof. For $x, y, z \in A$ and $\alpha, \beta \in \Omega$, by Eq. (31), we have $[x, x]_{\omega}=0$ and

$$
\begin{aligned}
{\left[p(x),[y, z]_{\alpha}\right]_{\beta}=} & {\left[p(x), y *_{\alpha} z-z *_{\alpha} y\right]_{\beta} } \\
= & p(x) *_{\beta}\left(y *_{\alpha} z-z *_{\alpha} y\right) \\
& -\left(y *_{\alpha} z-z *_{\alpha} y\right) *_{\beta} p(x) \\
= & p(x) *_{\beta}\left(y *_{\alpha} z\right)-p(x) *_{\beta}\left(z *_{\alpha} y\right) \\
& -\left(y *_{\alpha} z\right) *_{\beta} p(x)+\left(z *_{\alpha} y\right) *_{\beta} p(x) .
\end{aligned}
$$

Similarly, we have

$$
\begin{aligned}
{\left[p(y),[z, x]_{\alpha}\right]_{\beta}=} & p(y) *_{\beta}\left(z *_{\alpha} x\right)-p(y) *_{\beta}\left(x *_{\alpha} z\right) \\
& -\left(z *_{\alpha} x\right) *_{\beta} p(y)+\left(x *_{\alpha} z\right) *_{\beta} p(y), \\
{\left[p(z),[x, y]_{\alpha}\right]_{\beta}=} & p(z) *_{\beta}\left(x *_{\alpha} y\right)-p(z) *_{\beta}\left(y *_{\alpha} x\right) \\
& -\left(x *_{\alpha} y\right) *_{\beta} p(z)+\left(y *_{\alpha} x\right) *_{\beta} p(z), \\
{\left[p(x),[y, z]_{\beta}\right]_{\alpha}=} & p(x) *_{\alpha}\left(y *_{\beta} z\right)-p(x) *_{\alpha}\left(z *_{\beta} y\right) \\
& -\left(y *_{\beta} z\right) *_{\alpha} p(x)+\left(z *_{\beta} y\right) *_{\alpha} p(x), \\
{\left[p(y),[z, x]_{\beta}\right]_{\alpha}=} & p(y) *_{\alpha}\left(z *_{\beta} x\right)-p(y) *_{\alpha}\left(x *_{\beta} z\right) \\
& -\left(z *_{\beta} x\right) *_{\alpha} p(y)+\left(x *_{\beta} z\right) *_{\alpha} p(y), \\
{\left[p(z),[x, y]_{\beta}\right]_{\alpha}=} & p(z) *_{\alpha}\left(x *_{\beta} y\right)-p(z) *_{\alpha}\left(y *_{\beta} x\right) \\
& -\left(x *_{\beta} y\right) *_{\alpha} p(z)+\left(y *_{\beta} x\right) *_{\beta} p(z) .
\end{aligned}
$$

Then, by Eq. (30), we get

$$
\begin{aligned}
& {\left[p(x),[y, z]_{\alpha}\right]_{\beta}+\left[p(y),[z, x]_{\alpha}\right]_{\beta}+\left[p(z),[x, y]_{\alpha}\right]_{\beta}} \\
& \quad+\left[p(x),[y, z]_{\beta}\right]_{\alpha}+\left[p(y),[z, x]_{\beta}\right]_{\alpha}+\left[p(z),[x, y]_{\beta}\right]_{\alpha}=0 .
\end{aligned}
$$

Hence, $\left(A,[]_{\Omega}, p\right)$ is a compatible Hom-Lie algebra.

\section{Matching Rota-Baxter Algebras and Hom- Associative Algebras}

In this section, we extend the notion of matching Rota-Baxter algebras to the Hom-associative algebra setting.

Definition 14 [38]. Let $\lambda_{\Omega}:=\left(\lambda_{\omega}\right)_{\omega \in \Omega} \subseteq k$ be a set of scalars indexed by $\Omega$. A matching Rota-Baxter algebra of weight $\lambda_{\Omega}$ is an associative algebra $A$ equipped with a family $P_{\Omega}:=$ $\left(P_{\omega}\right)_{\omega \in \Omega}$ of linear operators $P_{\omega}: R \longrightarrow R, \omega \in \Omega$, that satisfy the matching Rota-Baxter equation

$$
\begin{aligned}
P_{\alpha}(x) \cdot P_{\beta}(y)= & P_{\alpha}\left(x \cdot P_{\beta}(y)\right)+P_{\beta}\left(P_{\alpha}(x) \cdot y\right) \\
& +\lambda_{\beta} P_{\alpha}(x \cdot y), \text { for all } x, y \in A \text { and } \alpha, \beta \in \Omega .
\end{aligned}
$$

Definition 15. A matching Hom-associative Rota-Baxter algebra is a quadruples $\left(A, \cdot, P_{\Omega}, p\right)$, where $\left(A, P_{\Omega}\right)$ is a matching 
Rota-Baxter algebra and $(A, \cdot, p)$ is a Hom-associative algebra.

Taking $p=\mathrm{i} d$, we recover to matching Rota-Baxter associative algebras and denote it by $\left(A, \cdot, P_{\Omega}\right)$. If $\Omega$ is a singleton, a matching Hom-associative Rota-Baxter algebra becomes a Hom-associative Rota-Baxter algebra given in [37].

A Hom-associative Rota-Baxter algebra can be induced from an associative Rota-Baxter algebra with a particular algebra endomorphism [37]. The following result generalizes it to the matching Rota-Baxter case.

Theorem 16. Let $\left(A, \cdot, P_{\Omega}\right)$ be a matching Rota-Baxter algebra and $p: A \longrightarrow A$ be an algebra endomorphism which commutes with $P_{\omega}$ for all $\omega \in \Omega$. Then $\left(A,{ }_{p}, P_{\Omega}, p\right)$, where $x_{p} y:=p(x \cdot y)$, is a matching Hom-associative Rota-Baxter algebra.

Proof. The Hom-associative structure of the algebra follows from Yau's Theorem in [52]. We only need to show that the matching Rota-Baxter equation holds. For $x, y \in A$ and $\alpha, \beta \in \Omega$,

$$
\begin{aligned}
P_{\alpha}(x) \cdot{ }_{p} P_{\beta}(y)= & \left.p\left(P_{\alpha}(x) \cdot P_{\beta}(y)\right) \text { by the definition of }{ }_{p}\right) \\
= & p\left(P_{\alpha}\left(x \cdot P_{\beta}(y)\right)+P_{\beta}\left(P_{\alpha}(x) \cdot y\right)\right. \\
& \left.+\lambda_{\beta} P_{\alpha}(x \cdot y)\right)(\text { by Eq. }(10)) \\
= & p\left(P_{\alpha}\left(x \cdot P_{\beta}(y)\right)\right)+p\left(P_{\beta}\left(P_{\alpha}(x) \cdot y\right)\right) \\
& +\lambda_{\beta} p\left(P_{\alpha}(x \cdot y)\right) \\
= & P_{\alpha}\left(p\left(x \cdot P_{\beta}(y)\right)\right)+P_{\beta}\left(p\left(P_{\alpha}(x) \cdot y\right)\right) \\
& +\lambda P_{\alpha}(p(x \cdot y))\left(\text { by } p \circ \mathrm{P}_{\omega}=\mathrm{P}_{\omega} \circ \mathrm{p}\right) \\
= & P_{\alpha}\left(x_{{ }_{p}} P_{\beta}(y)\right)+P_{\beta}\left(P_{\alpha}(x) \cdot{ }_{p} y\right)+\lambda P_{\alpha}\left(x_{p} y\right),
\end{aligned}
$$

as required.

Given a matching Hom-associative Rota-Baxter algebra $\left(A, \cdot, P_{\Omega}, p\right)$, it is natural to wonder that whether this matching Hom-associative Rota-Baxter algebra is induced by an ordinary associative matching Rota-Baxter algebra $\left(A, .^{\prime}\right.$, $\left.P_{\Omega}\right)$, i.e., $p$ is an algebra endomorphism with respect to .' and $\cdot=p \circ \cdot^{\prime}$.

Let $(A, \cdot, p)$ be a multiplicative Hom-associative algebra, i.e., $p(a \cdot b)=p(a) \cdot p(b)$ for all $a, b \in A$. It was proved in [53] that in case $p$ is invertible, $\left(A, p^{-1} \circ \cdot\right)$ is an associative algebra. It is generalized to the multiplicative Homassociative Rota-Baxter algebras in [37], and the following result generalizes it to the multiplicative matching Homassociative Rota-Baxter algebras.

Proposition 17. Let $\left(A, \cdot, P_{\Omega}, p\right)$ be a multiplicative matching Hom-assoicative Rota-Baxter algebra, where $p$ is invertible and $p \circ P_{\omega}=P_{\omega} \circ p$ for each $\omega \in \Omega$. Then, $\left(A, \cdot^{\prime}:=p^{-1} \circ, P_{\Omega}\right)$ is an associative matching Rota-Baxter algebra.
Proof. For $x, y, z \in A$, we have

$$
\begin{aligned}
\left(x \cdot{ }^{\prime} y\right) & { }^{\prime} z-x \cdot(y \cdot z) \\
= & p^{-1}\left(p^{-1}(x \cdot y) \cdot z\right)-p^{-1}\left(x \cdot p^{-1}(y \cdot z)\right)\left(\mathrm{by} \cdot{ }^{\prime}=\mathrm{p}^{-1} \circ \cdot\right) \\
= & p^{-2}((x \cdot y) \cdot p(z)-p(x) \cdot(y \cdot z)) \\
& \cdot(\operatorname{by} p(\mathrm{x}) \cdot \mathrm{p}(\mathrm{y})=\mathrm{p}(\mathrm{x} \cdot y))=0 .
\end{aligned}
$$

Hence, the associativity condition holds. For $\alpha, \beta \in \Omega$, we have

$$
\begin{aligned}
P_{\alpha}(x) \cdot^{\prime} P_{\beta}(y)= & p^{-1}\left(P_{\alpha}(x) \cdot P_{\beta}(y)\right) \\
= & p^{-1}\left(P_{\alpha}\left(x \cdot P_{\beta}(y)\right)+P_{\beta}\left(P_{\alpha}(x) \cdot y\right)\right. \\
& \left.+\lambda_{\beta} P_{\alpha}(x \cdot y)\right) \\
= & P_{\alpha}\left(p^{-1}\left(x \cdot P_{\beta}(y)\right)\right)+P_{\beta}\left(p^{-1}\left(P_{\alpha}(x) \cdot y\right)\right) \\
& +\lambda_{\beta} P_{\alpha}\left(p^{-1}(x \cdot y)\right) \\
= & P_{\alpha}\left(x \cdot{ }^{\prime} P_{\beta}(y)\right)+P_{\beta}\left(P_{\alpha}(x) \cdot{ }^{\prime} y\right) \\
& +\lambda_{\beta} P_{\alpha}(x \cdot y) .
\end{aligned}
$$

Hence, the matching Rota-Baxter equation holds for the new multiplication, and $\left(A, \cdot^{\prime}, P_{\Omega}\right)$ is an associative matching Rota-Baxter algebra.

There are two new ways of constructing Hom-associative algebras from a given multiplicative Hom-associative algebra $[37,54]$.

Definition 18. $([37,54])$. Let $(A, \cdot, p)$ be a multiplicative Homalgebra and $n \geq 0$. Then, the following two algebras are also Hom-associative algebras:

(a) the $n$-th derived Hom-algebra of type 1 of $A$ defined by

$$
A^{n}=\left(A,{ }^{(n)}=p^{n} \circ \cdot, p^{n+1}\right)
$$

(b) the $n$-th derived Hom-algebra of type 2 of $A$ defined by

$$
A^{n}=\left(A,{ }^{(n)}=p^{2^{n}-1} \circ \cdot, p^{2^{n}}\right)
$$

Now, we show that the $n$-th derived Hom-algebra of type 1 and 2 of a multiplicative matching Hom-associative RotaBaxter algebra is also a matching Hom-associative RotaBaxter algebra generalizing the Rota-Baxter case in [37]. 
Theorem 19. Let $\left(A, \cdot, P_{\Omega}, p\right)$ be a multiplicative matching Hom-associative Rota-Baxter algebra such that $p \circ P_{\omega}=P_{\omega} \circ$ $p$ for all $\omega \in \Omega$. Then,

(a) the $n$-th derived Hom-algebra of type 1 $\left(A,{ }^{(n)}=p^{n} \circ \cdot, p^{n+1}\right)$ is a matching Hom-associative Rota-Baxter algebra

(b) the $n$-th derived Hom-algebra of type $2\left(A, \cdot^{(n)}=\right.$ $\left.p^{2^{n}-1} \circ \cdot, p^{2^{n}}\right)$ is a matching Hom-associative RotaBaxter algebra

Proof. (a) By [54], $\left(A, \cdot^{n}, p^{n+1}\right)$ is a Hom-associative algebra. Now, we show the matching Rota-Baxter equation holds. For $x, y, z \in A$ and $\alpha, \beta \in \Omega$, we have

$$
\begin{aligned}
P_{\alpha}(x) \cdot{ }^{n} P_{\beta}(y)= & p^{n}\left(P_{\alpha}(x) \cdot P_{\beta}(y)\right)=p^{n}\left(P_{\alpha}\left(x \cdot P_{\beta}(y)\right)\right. \\
& \left.+P_{\beta}\left(P_{\alpha}(x) \cdot y\right)+\lambda_{\beta} P_{\alpha}(x \cdot y)\right) \\
= & P_{\alpha}\left(p^{n}\left(x \cdot P_{\beta}(y)\right)\right)+P_{\beta}\left(p^{n}\left(P_{\alpha}(x) \cdot y\right)\right) \\
& +\lambda_{\beta} P_{\alpha}\left(p^{n}(x \cdot y)\right)=P_{\alpha}\left(x \cdot{ }^{n} P_{\beta}(y)\right) \\
& +P_{\beta}\left(P_{\alpha}(x) \cdot{ }^{n} y\right)+\lambda_{\beta} P_{\alpha}\left(x \cdot{ }^{n} y\right)
\end{aligned}
$$

Thus, the matching Rota-Baxter equation holds for the new multiplication.

(b) By [54], $\left(A, \cdot^{(n)}=p^{2^{n}-1} \circ \cdot, p^{2^{n}}\right)$ is also a Homassociative algebra. For $x, y, z \in A$ and $\alpha, \beta \in \Omega$, we have

$$
\begin{aligned}
P_{\alpha}(x) \cdot{ }^{n} P_{\beta}(y)= & p^{2^{n}-1}\left(P_{\alpha}(x) \cdot P_{\beta}(y)\right)=p^{2^{n}-1}\left(P_{\alpha}\left(x \cdot P_{\beta}(y)\right)\right. \\
& \left.+P_{\beta}\left(P_{\alpha}(x) \cdot y\right)+\lambda_{\beta} P_{\alpha}(x \cdot y)\right) \\
= & P_{\alpha}\left(p^{2^{n}-1}\left(x \cdot P_{\beta}(y)\right)\right)+P_{\beta}\left(p^{2^{n}-1}\left(P_{\alpha}(x) \cdot y\right)\right) \\
& +\lambda_{\beta} P_{\alpha}\left(p^{2^{n}-1}(x \cdot y)\right)=P_{\alpha}\left(x \cdot{ }^{n} P_{\beta}(y)\right) \\
& +P_{\beta}\left(P_{\alpha}(x) \cdot{ }^{n} y\right)+\lambda_{\beta} P_{\alpha}\left(x \cdot{ }^{n} y\right) .
\end{aligned}
$$

This completes the proof.

Let $(A, \cdot)$ be an associative algebra. The centroid of $\mathrm{A}$ is defined by

$$
\begin{aligned}
\operatorname{Cent}(A): & :=\{p \in \operatorname{End}(A) \mid p(x \cdot y)=p(x) \cdot y \\
& =x \cdot p(y) \text { for all } x, y \in \mathrm{A}\}
\end{aligned}
$$

The same definition of the centroid is assumed for Homassociative algebras.

In [4], Benayadi and Makhlouf gave the construction of Hom-algebras using elements of the centroid for Lie algebras. In [37], the construction was extended to Rota-Baxter algebras. Now, we generalize it to the matching Rota-Baxter case.
Proposition 20. Let $\left(A, \cdot, P_{\Omega}\right)$ be an associative matching Rota-Baxter algebra. For $p \in \operatorname{Cent}(A)$ and $x, y \in A$, define

$$
x \cdot{ }_{p}^{1} y:=p(x) \cdot y \text { and } x \cdot{ }_{p}^{2} y:=p(x) \cdot p(y) \text {. }
$$

If $p \circ P_{\omega}=P_{\omega} \circ p$ for all $\omega \in \Omega$, then $\left(A,{ }_{p}^{1}, P_{\Omega}, p\right)$ and $\left(A,{ }_{p}^{2}, P_{\Omega}, p\right)$ are matching Hom-associative Rota-Baxter algebras.

Proof By [37]. $\left(A,{ }_{p}^{1}, p\right)$ and $\left(A, \cdot{ }_{p}^{2}, p\right)$ are Hom-associative algebras. Now, we show that they are also matching RotaBaxter algebras. For $x, y \in A$ and $\alpha, \beta \in \Omega$, we have

$$
\begin{aligned}
P_{\alpha}(x) \cdot{ }_{p}^{1} P_{\beta}(y)= & p\left(P_{\alpha}(x)\right) \cdot P_{\beta}(y)=P_{\alpha}(p(x)) \cdot P_{\beta}(y) \\
= & P_{\alpha}\left(p(x) \cdot P_{\beta}(y)\right)+P_{\beta}\left(P_{\alpha}(p(x)) \cdot y\right) \\
& +\lambda_{\beta} P_{\alpha}(p(x) \cdot y)=P_{\alpha}\left(x \cdot{ }_{p}^{1} P_{\beta}(y)\right) \\
& +P_{\beta}\left(P_{\alpha}(x) \cdot{ }_{p}^{1} y\right)+\lambda_{\beta} P_{\alpha}\left(x \cdot{ }_{p}^{1} y\right)
\end{aligned}
$$

and

$$
\begin{aligned}
P_{\alpha}(x) \cdot{ }_{p}^{2} P_{\beta}(y)= & p\left(P_{\alpha}(x)\right) \cdot p\left(P_{\beta}(y)\right)=P_{\alpha}(p(x)) \cdot P_{\beta}(p(y)) \\
= & P_{\alpha}\left(p(x) \cdot P_{\beta}(p(y))\right)+P_{\beta}\left(P_{\alpha}(p(x)) \cdot p(y)\right) \\
& +\lambda_{\beta} P_{\alpha}(p(x) \cdot p(y))=P_{\alpha}\left(p(x) \cdot p\left(P_{\beta}(y)\right)\right) \\
& +P_{\beta}\left(p\left(P_{\alpha}(x)\right) \cdot p(y)\right)+\lambda_{\beta} P_{\alpha}(p(x) \cdot p(y)) \\
= & P_{\alpha}\left(x \cdot{ }_{p}^{2} P_{\beta}(y)\right)+P_{\beta}\left(P_{\alpha}(x) \cdot{ }_{p}^{2} y\right)+\lambda_{\beta} P_{\alpha}\left(x \cdot{ }_{p}^{2} y\right) .
\end{aligned}
$$

This completes the proof.

\section{Matching Hom-Dendriform Algebras and Matching Hom-Tridendriform Algebras}

In this section, we introduce the notions of matching Homdendriform algebras and matching Hom-tridendriform algebras generalizing the definitions of matching dendriform algebras and matching tridendriform algebras given in [38].

Definition 21. A matching Hom-dendriform algebra is a kmodule $D$ together with a family of binary operations $\odot_{\omega}: D \otimes D \longrightarrow D$, where $\odot \in\{\prec, \succ\}$ and $\omega \in \Omega$, and a linear map $p: D \longrightarrow D$ such that for all $x, y, z \in D$ and $\alpha, \beta \in \Omega$,

$$
\begin{gathered}
\left(x \prec_{\alpha} y\right) \prec_{\beta} p(z)=p(x) \prec_{\alpha}\left(y \prec_{\beta} z\right)+p(x) \prec_{\beta}\left(y \succ_{\alpha} z\right), \\
\left(x \succ_{\alpha} y\right) \prec_{\beta} p(z)=p(x) \succ_{\alpha}\left(y \prec_{\beta} z\right), \\
\left(x \prec_{\beta} y\right) \succ_{\alpha} p(z)+\left(x \succ_{\alpha} y\right) \succ_{\beta} p(z)=p(x) \succ_{\alpha}\left(y \succ_{\beta} z\right) .
\end{gathered}
$$

For simplicity, we denote it by $\left(D, \prec_{\Omega}, \succ_{\Omega}, p\right)$.

Definition 22. A matching Hom-tridendriform algebra is a k-module $D$ together with a family of binary operations $\odot_{\omega}: D \otimes D \longrightarrow D$, where $\odot e \in\{\prec, \bullet, \succ\}$ and $\omega \in \Omega$, and a 
linear map $p: D \longrightarrow D$ such that for all $x, y, z \in D$ and $\alpha$, $\beta \in \Omega$,

$$
\begin{aligned}
\left(x \prec_{\alpha} y\right) \prec_{\beta} p(z)= & p(x) \prec_{\alpha}\left(y \prec_{\beta} z\right)+p(x) \prec_{\beta}\left(y \succ_{\alpha} z\right) \\
& +p(x) \prec_{\alpha}\left(y \bullet_{\beta} z\right), \\
\left(x \succ_{\alpha} y\right) \prec_{\beta} p(z)= & p(x) \succ_{\alpha}\left(y \prec_{\beta} z\right), \\
p(x) \succ_{\alpha}\left(y \succ_{\beta} z\right)= & \left(x \prec_{\beta} y\right) \succ_{\alpha} p(z)+\left(x \succ_{\alpha} y\right) \succ_{\beta} p(z) \\
& +\left(x \bullet_{\beta} y\right) \succ_{\alpha} p(z), \\
\left(x \succ_{\alpha} y\right) \bullet_{\beta} p(z)= & p(x) \succ_{\alpha}\left(y \bullet_{\beta} z\right), \\
\left(x \prec_{\alpha} y\right) \bullet_{\beta} p(z)= & p(x) \bullet_{\beta}\left(y \succ_{\alpha} z\right), \\
\left(x \bullet_{\alpha} y\right) \prec_{\beta} p(z)= & p(x) \bullet_{\alpha}\left(y<_{\beta} z\right), \\
\left(x \bullet_{\alpha} y\right) \bullet_{\beta} p(z)= & p(x) \bullet_{\alpha}\left(y \bullet_{\beta} z\right) .
\end{aligned}
$$

Definition 23.

(a) Let $\left(D,<_{\Omega},>_{\Omega}, p\right)$ and $\left(D^{\prime},<_{\Omega}^{\prime},>_{\Omega}^{\prime}, p^{\prime}\right)$ be two matching Hom-dendriform algebras. A linear map $f: D \longrightarrow D^{\prime}$ is called a matching Hom-dendriform algebra morphism if for all $\omega \in \Omega$

$\prec_{\omega}^{\prime} \circ(f \otimes f)=f \circ \prec_{\omega}, \succ_{\omega} \circ(f \otimes f)=f \circ \succ_{\omega}$ and $p^{\prime} \circ f=f \circ p$.

(b) Let $\left(D,<_{\Omega}, \bullet_{\Omega},>_{\Omega}, p\right)$ and $\left(D^{\prime},<_{\Omega}^{\prime}, \bullet_{\Omega},>_{\Omega}^{\prime}, p^{\prime}\right)$ be two matching Hom-tridendriform algebras. A linear map $f: D \longrightarrow D^{\prime}$ is called a matching Homtridendriform algebra morphism if for all $\omega \in \Omega$

$$
\begin{aligned}
\prec_{\omega}^{\prime} \circ(f \otimes f) & =f \circ \prec_{\omega}, \bullet_{\omega}^{\prime} \circ(f \otimes f)=f \circ \bullet_{\omega}^{\prime}, \succ_{\omega} \circ(f \otimes f) \\
& =f \circ \succ_{\omega} \text { and } p^{\prime} \circ f=f \circ p .
\end{aligned}
$$

The following results show that we can construct a matching Hom-(tri)dendriform algebra from a matching (tri)dendriform algebra, generalizing the (tri)dendriform case in [37].

\section{Theorem 24.}

(a) Let $\left(D, \prec_{\Omega},>_{\Omega}\right)$ be a matching dendriform algebra and $p: D \longrightarrow D$ be a matching dendriform algebra endomorphism. Then, $A_{p}=\left(A,<_{p, \Omega}, \succ_{p, \Omega}, p\right)$, where $<_{p, \omega}:=p \circ \prec_{\omega}$ and $\succ_{p, \omega}:=p \circ \succ_{\omega}$ for each $\omega \in \Omega$, is a matching Hom-dendriform algebra. Moreover, suppose that $\left(A^{\prime},<_{\Omega}^{\prime}, \succ_{\Omega}^{\prime}\right)$ is another matching dendriform algebra and $p^{\prime}: A^{\prime} \longrightarrow A^{\prime}$ is a matching dendriform algebra endomorphism. If $f: A \longrightarrow A^{\prime}$ is a matching dendriform algebra morphism that satisfies $f \circ p=p^{\prime} \circ f$, then

$$
f:\left(D, \prec_{p, \Omega},>_{p, \Omega}, p\right) \longrightarrow\left(D^{\prime}, \prec_{p, \Omega}^{\prime}, \succ_{p, \Omega}^{\prime}, p^{\prime}\right)
$$

is a morphism of matching Hom-dendriform algebras.

(b) Let $\left(D,<_{\Omega}, \bullet_{\Omega},>_{\Omega}\right)$ be a matching tridendriform algebra and $p: D \longrightarrow D$ be a matching tridendriform algebra endomorphism. Then, $A_{p}=\left(A,<_{p, \Omega}, \bullet_{p, \Omega}\right.$, $\left.>_{p, \Omega}, p\right)$, where $<_{p, \omega}:=p \circ \prec_{\omega}, \bullet_{p, \omega}:=p \circ \bullet_{\omega}$ and $\succ_{p, \omega}=$ $p \circ>_{\omega}$ for each $\omega \in \Omega$, is a matching Homtridendriform algebra. Moreover, suppose that $\left(A^{\prime}\right.$, $\left.\left.<_{\Omega}^{\prime}, \bullet_{\Omega}^{\prime},\right\rangle_{\Omega}^{\prime}\right)$ is another matching tridendriform algebra and $p^{\prime}: A^{\prime} \longrightarrow A^{\prime}$ is a matching tridendriform algebra endomorphism. If $f: A \longrightarrow A^{\prime}$ is a matching tridendriform algebra morphism that satisfies $f \circ p=$ $p^{\prime} \circ f$, then

$f:\left(D, \prec_{p, \Omega}, \bullet_{p, \Omega}, \succ_{p, \Omega}, p\right) \longrightarrow\left(D^{\prime}, \prec_{p, \Omega}^{\prime}, \bullet_{p, \Omega}^{\prime}, \succ_{p, \Omega}^{\prime}, p^{\prime}\right)$

is a morphism of matching Hom-tridendriform algebras.

Proof. We just prove Item (b) and Item (a) can be proved similarly. For any $x, y, z \in A$ and $\alpha, \beta \in \Omega$, we have

$$
\begin{aligned}
& \left(x \prec_{p, \alpha} y\right) \prec_{p, \beta} p(z)=p\left(p\left(x \prec_{\alpha} y\right) \prec_{\beta} p(z)\right)=p^{2}\left(\left(x \prec_{\alpha} y\right) \prec_{\beta} z\right) ; \\
& p(x) \prec_{p, \alpha}\left(y \prec_{p, \beta} z\right)=p\left(p(x) \prec_{\alpha} p\left(y \prec_{\beta} z\right)\right)=p^{2}\left(x \prec_{\alpha}\left(y \prec_{\beta} z\right)\right) ; \\
& p(x) \prec_{p, \beta}\left(y \succ_{p, \alpha} z\right)=p\left(p(x) \prec_{\beta} p\left(y \succ_{\alpha} z\right)\right)=p^{2}\left(x \prec_{\beta}\left(y \succ_{\alpha} z\right)\right) ; \\
& p(x) \prec_{p, \alpha}\left(y \bullet_{p, \beta} z\right)=p\left(p(x) \prec_{\alpha} p\left(y \bullet_{\beta} z\right)\right)=p^{2}\left(x \prec_{\alpha}\left(y \bullet_{\beta} z\right)\right) .
\end{aligned}
$$

Hence,

$$
\begin{aligned}
\left(x<_{p, \alpha} y\right) \prec_{p, \beta} p(z)= & p(x) \prec_{p, \alpha}\left(y \prec_{p, \beta} z\right)+p(x) \prec_{p, \beta}\left(y \succ_{p, \alpha} z\right) \\
& +p(x) \prec_{p, \alpha}\left(y \bullet_{p, \beta} z\right),
\end{aligned}
$$

that is Eq. (48) holds for $\left(A, \prec_{p, \Omega}, \bullet_{p, \Omega},>_{p, \Omega}, p\right)$. Similarly, Eqs. (49), (50), (51), (52), (53), (54) hold. Hence, $\left(A,<_{p, \Omega}, \bullet_{p, \Omega}\right.$, $\left.\succ_{p, \Omega}, p\right)$ is a matching Hom-tridendriform algebra. And

$$
\begin{aligned}
f(x) \prec_{p}{ }^{\prime}, \alpha f(y) & =p^{\prime}\left(f(x) \prec_{\alpha} f(y)\right)=p^{\prime} \circ f\left(x \prec_{\alpha} y\right) \\
& =f \circ p\left(x \prec_{\alpha} y\right)=f\left(x \prec_{p, \alpha} y\right) ; \\
f(x) \succ_{p}{ }^{\prime}, \alpha f(y) & =p^{\prime}\left(f(x) \succ_{\alpha} f(y)\right)=p^{\prime} \circ f\left(x \succ_{\alpha} y\right) \\
& =f \circ p\left(x \succ_{\alpha} y\right)=f\left(x \succ_{p, \alpha} y\right) ; \\
f(x) \bullet_{p}{ }^{\prime}, \alpha f(y) & =p^{\prime}\left(f(x) \bullet_{\alpha} f(y)\right)=p^{\prime} \circ f\left(x \bullet_{\alpha}(y)\right) \\
& =f \circ p(x \bullet y)=f\left(x \bullet_{p, \alpha} y\right) .
\end{aligned}
$$


Hence, $f:\left(D, \prec_{p, \Omega}, \bullet_{p, \Omega},>_{p, \Omega}, p\right) \longrightarrow\left(D^{\prime}, \prec_{p, \Omega}^{\prime}, \bullet_{p, \Omega}^{\prime},>_{p, \Omega}^{\prime}, p^{\prime}\right)$ is a morphism of matching Hom-tridendriform algebras.

Now, we show that any linear combinations of the operations of a matching Hom-dendriform algebra still result in a matching Hom-dendriform algebra, generalizing the matching dendriform case in [38].

Proposition 25. Let I be an nonempty set. For each $i \in I$, let $A_{i}: \Omega \longrightarrow k$ be a map with finite supports, identified with finite set $A_{i}=\left(a_{i, \omega}\right)_{\omega \in \Omega}, a_{i, \omega} \in k$.

(a) Let $\left(D, \prec_{\Omega}, \succ_{\Omega}, p\right)$ be a matching Hom-dendriform algebra. Define the following binary operations:

$$
\odot_{i}:=\sum_{\omega \in \Omega} a_{i, \omega} \odot, \text { where } \odot \in\{<, \succ\} \text { and } i \in I \text {. }
$$

Then, $\left(D, \prec_{I}, \succ_{I}, p\right)$ is also a matching Homdendriform algebra.

(b) Let $\left(T,<_{\Omega}, \bullet_{\Omega}, \succ_{\Omega}, p\right)$ be a matching Homtridendriform algebra. Define the following binary operations:

$\odot_{i}:=\sum_{\omega \in \Omega} a_{i, \omega} \odot_{\omega}$ where $\odot \in\{\prec, \bullet,>\}$ and $i \in I$.

Then, $\left(T, \prec_{I}, \bullet_{I}, \succ_{I}, p\right)$ is also a matching Homtridendriform algebra.

Proof. We just prove Item (b) and Item (a) can be proved similarly. For $x, y, z \in D$ and $i, j \in I$, we have

$$
\begin{aligned}
\left(x \prec_{i} y\right) \prec_{j} p(z)= & \sum_{\beta \in \Omega} b_{j, \beta}\left(\sum_{\alpha \in \Omega} a_{i, \alpha} x \prec_{\alpha} y\right) \prec_{\beta} p(z) \\
= & \sum_{\alpha \in \Omega} \sum_{\beta \in \Omega} a_{i, \alpha} b_{j, \beta}\left(x \prec_{\alpha} y\right) \prec_{\beta} p(z) \\
= & \sum_{\alpha \in \Omega} \sum_{\beta \in \Omega} a_{i, \alpha} b_{j, \beta}\left(p(x) \prec_{\alpha}\left(y \prec_{\beta} z\right)\right. \\
& \left.+p(x) \prec_{\beta}\left(y \succ_{\alpha} z\right)+p(x) \prec_{\alpha}\left(y \bullet_{\beta} z\right)\right) \\
= & \sum_{\alpha \in \Omega} a_{i, \alpha} p(x) \prec_{\alpha}\left(\sum_{\beta \in \Omega} b_{j, \beta} y \prec_{\beta} z\right) \\
& +\sum_{\beta \in \Omega} b_{j, \beta} p(x) \prec_{\beta}\left(\sum_{\alpha \in \Omega} a_{i, \alpha} y \succ_{\alpha} z\right) \\
& +\sum_{\alpha \in \Omega} a_{i, \alpha} p(x) \prec_{\alpha}\left(\sum_{\beta \in \Omega} b_{j, \beta} y \bullet_{\beta} z\right)
\end{aligned}
$$

$$
\begin{aligned}
= & \sum_{\alpha \in \Omega} a_{i, \alpha} p(x) \prec_{\alpha}\left(y \prec_{j} z\right)+\sum_{\beta \in \Omega} b_{j, \beta} p(x) \prec_{\beta}\left(y \succ_{i} z\right) \\
& +\sum_{\alpha \in \Omega} a_{i, \alpha} p(x) \prec_{\alpha}\left(y \bullet_{j} z\right) \\
= & p(x) \prec_{i}\left(y \prec_{j} z\right)+p(x) \prec_{j}\left(y \succ_{i} z\right)+p(x) \prec_{i}\left(y \bullet_{j} z\right) .
\end{aligned}
$$

Hence, Eq. (48) holds. Similarly, Eqs. (49), (50), (51), (52), (53), (54) hold. Hence, $\left(T, \prec_{I}, \bullet_{I}, \succ_{I}, p\right)$ is a matching Hom-tridendriform algebra.

The following results establish the connections between matching Hom-(tri)dendriform algebras and compatible Hom-associative algebras, generalizing the well-known result that a (tri) dendriform algebra has an associative algebraic structure.

\section{Theorem 26.}

(a) Let $\left(A,<_{\Omega}, \succ_{\Omega}, p\right)$ be a matching Hom-dendriform algebra. Then $\left(A, \cdot_{\Omega}, p\right)$ is a compatible Homassociative algebra, where

${ }_{\omega}: A \otimes A \longrightarrow A, x \cdot{ }_{\omega} y:=x \prec_{\omega} y+x \succ_{\omega} y$ for $x, y \in A$ and $\omega \in \Omega$.

(b) Let $\left(A,<_{\Omega}, \bullet_{\Omega},>_{\Omega}, p\right)$ be a matching Homtridendriform algebra. Then, $(A, \cdot \Omega, p)$ is a compatible Hom-associative algebra, where

$$
\begin{aligned}
{ }_{\omega}: A \otimes A \longrightarrow A, x \cdot{ }_{\omega} y & :=x \prec_{\omega} y+x \bullet_{\omega} y+x \succ_{\omega} y \text { for } x, y \\
& \in A \text { and } \omega \in \Omega .
\end{aligned}
$$

Proof. We only prove Item (b) and Item (a) can be proved similarly. For $x, y, z \in A$ and $\alpha, \beta \in \Omega$, we have

$$
\begin{aligned}
& \left(x \cdot{ }_{\alpha} y\right) \cdot{ }_{\beta} p(z)+\left(x \cdot{ }_{\beta} y\right) \cdot{ }_{\alpha} p(z) \\
& =\left(x \prec_{\alpha} y+x \bullet_{\alpha} y+x \succ_{\alpha} y\right) \cdot{ }_{\beta} p(z)+\left(x<_{\beta} y+x \bullet_{\beta} y+x \succ_{\beta} y\right) \cdot{ }_{\alpha} p(z) \\
& =\left(x<_{\alpha} y\right)<_{\beta} p(z)+\left(x \bullet_{\alpha} y\right)<_{\beta} p(z)+\left(x \succ_{\alpha} y\right)<_{\beta} p(z) \\
& +\left(x \prec_{\alpha} y\right) \bullet_{\beta} p(z)+\left(x \bullet_{\alpha} y\right) \bullet \bullet_{\beta} p(z)+\left(x \succ_{\alpha} y\right) \bullet_{\beta} p(z) \\
& +\left(x<_{\alpha} y\right) \succ_{\beta} p(z)+\left(x \bullet_{\alpha} y\right)>_{\beta} p(z)+\left(x \succ_{\alpha} y\right)>_{\beta} p(z) \\
& +\left(x<_{\beta} y\right) \prec_{\alpha} p(z)+\left(x \succ_{\beta} y\right) \prec_{\alpha} p(z)+\left(x \bullet_{\beta} y\right)<_{\alpha} p(z) \\
& +\left(x<_{\beta} y\right) \bullet_{\alpha} p(z)+\left(x \succ_{\beta} y\right) \bullet_{\alpha} p(z)+\left(x \bullet_{\beta} y\right) \bullet_{\alpha} p(z) \\
& +\left(x<_{\beta} y\right)>_{\alpha} p(z)+\left(x \bullet_{\beta} y\right)>_{\alpha} p(z)+\left(x>_{\beta} y\right)>_{\alpha} p(z),
\end{aligned}
$$




$$
\begin{aligned}
& p(x) \cdot{ }_{\alpha}\left(y \cdot{ }_{\beta} z\right)+p(x) \cdot{ }_{\beta}\left(y \cdot{ }_{\alpha} z\right) \\
& =p(x) \cdot{ }_{\alpha}\left(y \prec_{\beta} z+y \bullet_{\beta} z+y \succ_{\beta} z\right)+p(x) \cdot_{\beta}\left(y<_{\alpha} z+y \bullet_{\alpha} z+y \succ_{\alpha} z\right) \\
& =p(x) \prec_{\alpha}\left(y \prec_{\beta} z\right)+p(x) \prec_{\alpha}\left(y \bullet_{\beta} z\right)+p(x) \prec_{\alpha}\left(y \succ_{\beta} z\right) \\
& +p(x) \bullet_{\alpha}\left(y<_{\beta} z\right)+p(x) \bullet_{\alpha}\left(y \bullet_{\beta} z\right)+p(x) \bullet_{\alpha}\left(y \succ_{\beta} z\right) \\
& +p(x)>_{\alpha}\left(y \prec_{\beta} z\right)+p(x)>_{\alpha}\left(y \bullet_{\beta} z\right)+p(x)>_{\alpha}\left(y>_{\beta} z\right) \\
& +p(x) \prec_{\beta}\left(y \prec_{\alpha} z\right)+p(x) \prec_{\beta}\left(y \bullet_{\alpha} z\right)+p(x) \prec_{\beta}\left(y \succ_{\alpha} z\right) \\
& +p(x) \bullet_{\beta}\left(y \prec_{\alpha} z\right)+p(x) \bullet_{\beta}\left(y \bullet_{\alpha} z\right)+p(x) \bullet_{\beta}\left(y \succ_{\alpha} z\right) \\
& +p(x)>_{\beta}\left(y \prec_{\alpha} z\right)+p(x)>_{\beta}\left(y \bullet_{\alpha} z\right)+p(x)>_{\beta}\left(y \succ_{\alpha} z\right) .
\end{aligned}
$$

By Eqs (48), (49), (50), (51), (52), (53), (54), we get

$$
\left(x \cdot{ }_{\alpha} y\right) \cdot{ }_{\beta} p(z)+\left(x \cdot_{\beta} y\right) \cdot{ }_{\alpha} p(z)=p(x) \cdot{ }_{\alpha}\left(y \cdot{ }_{\beta} z\right)+p(x) \cdot{ }_{\beta}\left(y \cdot{ }_{\alpha} z\right) .
$$

Hence, $\left(A,{ }_{\Omega}, p\right)$ is a compatible Hom-associative algebra.

Now, we explore the relationship between matching Hom-dendriform algebras and matching Hom-preLie algebras.

Theorem 27. Let $\left(A, \prec_{\Omega}, \succ_{\Omega}, p\right)$ be a matching Homdendriform algebra. Then $\left(A, *_{\Omega}, p\right)$ is a matching HompreLie algebra, where

$*_{\omega}: A \otimes A \longrightarrow A, x *_{\omega} y:=x \succ_{\omega} y-y \prec_{\omega} x$ for $x, y \in A$ and $\omega \in \Omega$.

Proof. For $x, y, z \in A$ and $\alpha, \beta \in \Omega$, we have

$$
\begin{aligned}
p(x) *_{\alpha}\left(y *_{\beta} z\right)-\left(x *_{\alpha} y\right) *_{\beta} p(z) \\
=p(x) *_{\alpha}\left(y \succ_{\beta} z-z \prec_{\beta} y\right)-\left(x \succ_{\alpha} y-y \prec_{\alpha} x\right) *_{\beta} p(z) \\
=p(x) \succ_{\alpha}\left(y \succ_{\beta} z\right)-p(x) \succ_{\alpha}\left(z \prec_{\beta} y\right)-\left(y \succ_{\beta} z\right) \prec_{\alpha} p(x) \\
\quad+\left(z \prec_{\beta} y\right) \prec_{\alpha} p(x)-\left(x \succ_{\alpha} y\right) \succ_{\beta} p(z)+\left(y \prec_{\alpha} x\right) \succ_{\beta} p(z) \\
\quad+p(z) \prec_{\beta}\left(x \succ_{\alpha} y\right)-p(z) \prec_{\beta}\left(y \prec_{\alpha} x\right)
\end{aligned}
$$

and

$$
\begin{aligned}
& p(y) *_{\beta}\left(x *_{\alpha} z\right)-\left(y *_{\beta} x\right) *_{\alpha} p(z) \\
&= p(y) *_{\beta}\left(x \succ_{\alpha} z-z \prec_{\alpha} x\right)-\left(y \succ_{\beta} x-x \prec_{\beta} y\right) *_{\alpha} p(z) \\
&= p(y) \succ_{\beta}\left(x \succ_{\alpha} z\right)-p(y) \succ_{\beta}\left(z \prec_{\alpha} x\right)-\left(x \succ_{\alpha} z\right) \prec_{\beta} p(y) \\
&+\left(z \prec_{\alpha} x\right) \prec_{\beta} p(y)-\left(y \succ_{\beta} x\right) \succ_{\alpha} p(z)+\left(x \prec_{\beta} y\right) \succ_{\alpha} p(z) \\
&+p(z) \prec_{\alpha}\left(y \succ_{\beta} x\right)-p(z) \prec_{\alpha}\left(x \prec_{\beta} y\right) .
\end{aligned}
$$

By Eqs (48), (49), (50), (51), (52), (53), (54), we get

$$
\begin{aligned}
& p(x) *_{\alpha}\left(y *_{\beta} z\right)-\left(x *_{\alpha} y\right) *_{\beta} p(z) \\
& \quad=p(y) *_{\beta}\left(x *_{\alpha} z\right)-\left(y *_{\beta} x\right) *{ }_{\alpha} p(z) .
\end{aligned}
$$

Hence, $\left(A, *_{\Omega}, p\right)$ is a matching Hom-preLie algebra.

A matching Rota-Baxter algebra $\left(A, \cdot, P_{\Omega}\right)$ is of weight 0 if the set $\lambda_{\Omega}=\{0\}$. The connections between Rota-Baxter algebras and (tri)dendriform algebras are given in $[36,41]$ and extended to matching Rota-Baxter algebras. Now, we generalize it to matching Hom-associative Rota-Baxter algebra.

\section{Proposition 28.}

(a) Let $\left(A, \cdot, P_{\Omega}, p\right)$ be a matching Hom-associative RotaBaxter algebra of weight 0 . Assume that $p \circ P_{\omega}=P_{\omega}$ $\circ p$ for each $\omega \in \Omega$. Define the operations $\prec_{\omega}$ and $\succ_{\omega}$ for $\omega \in \Omega$ by

$$
x \prec_{\omega} y:=x \cdot P_{\omega}(y) \text { and } x \succ_{\omega} y=P_{\omega}(x) \cdot y, \text { for } x, y \in A .
$$

Then $\left(A, \prec_{\Omega}, \succ_{\Omega}, p\right)$ is a matching Hom-dendriform algebra.

(b) Let $\left(A, \cdot, P_{\Omega}, p\right)$ be a matching Hom-associative RotaBaxter algebra. Assume that $p \circ P_{\omega}=P_{\omega} \circ p$ for each $\omega \in \Omega$. Define the operations $\prec_{\omega}, \succ_{\omega}, \omega \in \Omega$ by

$x \prec_{\omega} y:=x \cdot P_{\omega}(y)+\lambda_{\omega} x \cdot y$ and $x \succ_{\omega} y=P_{\omega}(x) \cdot y$, for $x, y \in A$.

Then, $\left(A, \prec_{\Omega}, \succ_{\Omega}, p\right)$ is a matching Hom-dendriform algebra.

Proof. Since Item (a) can be seen as a special case of Item (b) by taking $\lambda_{\Omega}=\{0\}$, we only prove Item (b). For $x, y, z \in A$ and $\alpha, \beta \in \Omega$, we have

$$
\begin{aligned}
& p(x) \prec_{\alpha}\left(y \prec_{\beta} z\right)+p(x) \prec_{\beta}\left(y \succ_{\alpha} z\right) \\
&= p(x) \prec_{\alpha}\left(y \cdot P_{\beta}(z)+\lambda_{\beta} y \cdot z\right)+p(x) \prec_{\beta}\left(P_{\alpha}(y) \cdot z\right) \\
&= p(x) \cdot P_{\alpha}\left(y \cdot P_{\beta}(z)+\lambda_{\beta} y \cdot z\right)+\lambda_{\alpha} p(x) \\
& \cdot\left(y \cdot P_{\beta}(z)+\lambda_{\beta} y \cdot z\right)+p(x) \cdot P_{\beta}\left(P_{\alpha}(y) \cdot z\right) \\
&+\lambda_{\beta} p(x) \cdot\left(P_{\alpha}(y) \cdot z\right)=p(x)\left(P_{\alpha}(y) \cdot P_{\beta}(z)\right) \\
&+\lambda_{\alpha} p(x) \cdot\left(y \cdot P_{\beta}(z)\right)+\lambda_{\alpha} \lambda_{\beta} p(x) \cdot(y \cdot z) \\
&+\lambda_{\beta} p(x) \cdot\left(P_{\alpha}(y) \cdot z\right)=\left(x \cdot P_{\alpha}(y)+\lambda_{\alpha} x \cdot y\right) \\
& \cdot P_{\beta}(p(z))+\lambda_{\beta}\left(x \cdot P_{\alpha}(y)+\lambda_{\alpha} x \cdot y\right) \cdot p(z) \\
&=\left(x \cdot P_{\alpha}(y)+\lambda_{\alpha} x \cdot y\right) \prec_{\beta} p(z)=\left(x \prec_{\alpha} y\right) \prec_{\beta} p(z) .
\end{aligned}
$$


Also,

$$
\begin{aligned}
\left(x \succ_{\alpha} y\right) \prec_{\beta} p(z) & =\left(P_{\alpha}(x) \cdot y\right) \prec_{\beta} p(z) \\
& =\left(P_{\alpha}(x) \cdot y\right) \cdot P_{\beta}(p(z))+\lambda_{\beta}\left(P_{\alpha}(x) \cdot y\right) \cdot p(z) \\
& =P_{\alpha}(p(x)) \cdot\left(y \cdot P_{\beta}(z)\right)+\lambda_{\beta} P_{\alpha}(p(x)) \cdot(y \cdot z) \\
& =P_{\alpha}(p(x)) \cdot\left(y \cdot P_{\beta}(z)+\lambda_{\beta} y \cdot z\right) \\
& =P_{\alpha}(p(x)) \cdot\left(y \prec_{\beta} z\right)=p(x) \succ_{\alpha}\left(y \prec_{\beta} z\right)
\end{aligned}
$$

and

$$
\begin{aligned}
(x & \left.{ }_{\beta} y\right) \succ_{\alpha} p(z)+\left(x \succ_{\alpha} y\right)>_{\beta} p(z) \\
& =\left(x \cdot P_{\beta}(y)+\lambda_{\beta} x \cdot y\right) \succ_{\alpha} p(z)+\left(P_{\alpha}(x) \cdot y\right)>_{\beta} p(z) \\
& =P_{\alpha}\left(x \cdot P_{\beta}(y)+\lambda_{\beta} x \cdot y\right) \cdot p(z)+P_{\beta}\left(P_{\alpha}(x) \cdot y\right) \cdot p(z) \\
& =\left(P_{\alpha}\left(x \cdot P_{\beta}(y)\right)+P_{\beta}\left(P_{\alpha}(x) \cdot y\right)+\lambda_{\beta} P_{\alpha}(x \cdot y)\right) \cdot p(z) \\
& =\left(P_{\alpha}(x) \cdot P_{\beta}(y)\right) \cdot p(z)=P_{\alpha}(p(x)) \cdot\left(P_{\beta}(y) \cdot z\right) \\
& =p(x)>_{\alpha}\left(y \succ_{\beta} z\right) .
\end{aligned}
$$

Hence, $\left(A, \prec_{\Omega}, \succ_{\Omega}, p\right)$ is a matching Hom-dendriform algebra.

Proposition 29. Let $\left(A, \cdot, P_{\Omega}, p\right)$ be a matching Homassociative Rota-Baxter algebra. Assume that $p \circ P_{\omega}=P_{\omega} \circ$ $p$ for each $\omega \in \Omega$. Define the operations $\prec_{\omega}$, $\rangle_{\omega}$ and $\bullet_{\omega}$ for $\omega \in \Omega$ by

$$
\begin{aligned}
& x \prec_{\omega} y:=x \cdot P_{\omega}(y), x \succ_{\omega} y=P_{\omega}(x) \cdot y \text { and } \\
& x \bullet_{\omega} y=\lambda_{\omega} x \cdot y, \text { for } x, y \in A .
\end{aligned}
$$

Then, $\left(A, \prec_{\Omega}, \bullet_{\Omega},>_{\Omega}, p\right)$ is a matching Homtridendriform algebra.

Proof. For $x, y, z \in A$ and $\alpha, \beta \in \Omega$, we have

$$
\begin{aligned}
\left(x \prec_{\alpha} y\right) \prec_{\beta}(p(z))= & \left(x \cdot P_{\alpha}(y)\right) \cdot P_{\beta}(p(z))=p(x) \cdot\left(P_{\alpha}(y) \cdot P_{\beta}(z)\right) \\
= & p(x) \cdot\left(P_{\alpha}\left(y \cdot P_{\beta}(z)\right)+P_{\beta}\left(P_{\alpha}(y) \cdot z\right)\right. \\
& \left.+\lambda_{\beta} P_{\alpha}(y \cdot z)\right)=p(x) \prec_{\alpha}\left(y \prec_{\beta}(z)\right) \\
& +p(x) \prec_{\beta}\left(y \succ_{\alpha} z\right)+x \prec_{\alpha}\left(y \bullet_{\beta} z\right),
\end{aligned}
$$

$$
\begin{aligned}
\left(x \succ_{\alpha} y\right)<_{\beta} p(z)= & \left(P_{\alpha}(x) \cdot y\right) \cdot P_{\beta}(p(z))=P_{\alpha}(p(x)) \cdot\left(y \cdot P_{\beta}(z)\right) \\
= & p(x) \succ_{\alpha}\left(y<_{\beta} z\right), \\
p(x) \succ_{\alpha}\left(y \succ_{\beta} z\right)= & P_{\alpha}(p(x)) \cdot\left(P_{\beta}(y) \cdot z\right)=\left(P_{\alpha}(x) \cdot P_{\beta}(y)\right) \cdot p(z) \\
= & \left(P_{\alpha}\left(x \cdot P_{\beta}(y)\right)+P_{\beta}\left(P_{\alpha}(x) \cdot y\right)\right. \\
& \left.+\lambda_{\beta} P_{\alpha}(x \cdot y)\right) \cdot p(z)=\left(x \prec_{\beta} y\right) \succ_{\alpha} p(z) \\
& +\left(x \succ_{\alpha} y\right)>_{\beta} p(z)+\left(x \bullet_{\beta} y\right) \succ_{\alpha} p(z),
\end{aligned}
$$$$
\left(x \succ_{\alpha} y\right) \bullet{ }_{\beta} p(z)=\lambda_{\beta}\left(P_{\alpha}(x) \cdot y\right) \cdot p(z)=\lambda_{\beta} P_{\alpha}(p(x)) \cdot(y \cdot z)
$$$$
=p(x)>_{\alpha}\left(y \bullet_{\beta} z\right) \text {, }
$$

$$
\begin{aligned}
\left(x<_{\alpha} y\right) \bullet_{\beta} p(z) & =\lambda_{\beta}\left(x \cdot P_{\alpha}(y)\right) \cdot p(z)=\lambda_{\beta} p(x) \cdot\left(P_{\alpha}(y) \cdot z\right) \\
& =p(x) \bullet_{\beta}\left(y \succ_{\alpha} z\right), \\
\left(x \bullet_{\alpha} y\right) \prec_{\beta} p(z) & =\lambda_{\alpha}(x \cdot y) \cdot P_{\beta}(p(z))=\lambda_{\alpha} p(x) \cdot\left(y \cdot P_{\beta}(z)\right) \\
& =p(x) \bullet_{\alpha}\left(y<_{\beta} z\right), \\
\left(x \bullet_{\alpha} y\right) \bullet_{\beta} p(z) & =\lambda_{\alpha} \lambda_{\beta}(x \cdot y) \cdot p(z)=\lambda_{\alpha} \lambda_{\beta} p(x) \cdot(y \cdot z) \\
& =p(x) \bullet_{\alpha}\left(y \bullet_{\beta} z\right),
\end{aligned}
$$

as required.

\section{Corollary 30.}

(a) Let $\left(A, \cdot, P_{\Omega}, p\right)$ be a matching Hom-associative RotaBaxter algebra of weight 0 . Then, $\left(A, *_{\Omega}\right)$ is a matching Hom-preLie algebra, where

$$
x *{ }_{\omega} y:=P_{\omega}(x) \cdot y-y \cdot P_{\omega}(x) \text { for } x, y \in A \text { and } \omega \in \Omega .
$$

(b) Let $\left(A, \cdot, P_{\Omega}, p\right)$ be a matching Hom-associative RotaBaxter algebra. Then, $\left(A, *_{\Omega}\right)$ is a matching HompreLie algebra, where

$x *_{\omega} y:=P_{\omega}(x) \cdot y-y \cdot P_{\omega}(x)-\lambda_{\omega} y \cdot x$ for $x, y \in A$ and $\omega \in \Omega$.

Proof. (a) It follows from Theorem 27 and Proposition 28 (a). (b) It follows from Theorem 27 and Proposition 28 (b).

\section{Matching Rta-Baxter Operators and Hom- Nonassociative Algebras}

Rota-Baxter Lie algebras were introduced independently by Belavin and Drinfeld and Semenov-Tian-Shansky in [51, 55] and were related to solutions of the (modified) YangBaxter equation. Makhlouf extended Rota-Baxter operators to the context of Hom-Lie algebras. Now, we generalize it to the matching Rota-Baxter case.

Definition 31. Let $\lambda_{\Omega}:=\left(\lambda_{\omega}\right)_{\omega \in \Omega} \subseteq k$ be a family indexed by $\Omega$. A matching Hom-Lie Rota-Baxter algebra is a Hom-Lie algebra $(\mathfrak{g},[], p)$ endowed with a set of linear maps $P_{\omega}: \mathfrak{g} \longrightarrow \mathfrak{g}$, where $\omega \in \Omega$, subject to the relation

$$
\begin{aligned}
{\left[P_{\alpha}(x), P_{\beta}(y)\right]=} & P_{\alpha}\left(\left[x, P_{\beta}(y)\right]\right)+P_{\beta}\left(\left[P_{\alpha}(x), y\right]\right) \\
& +\lambda_{\beta} P_{\alpha}([x, y]),
\end{aligned}
$$

for all $x, y \in \mathfrak{g}$ and $\alpha, \beta \in \Omega$. For simplicity, we denote it by $\left(\mathfrak{g},[], P_{\Omega}, p\right)$.

Theorem 32. Let $\left(\mathfrak{g},[], P_{\Omega}\right)$ be a matching Lie Rota-Baxter algebra and $p: \mathfrak{g} \longrightarrow \mathfrak{g}$ be a Lie algebra endomorphism such 
that $p \circ P_{\omega}=P_{\omega} \circ p$ for each $\omega \in \Omega$. Then, $\left(\mathfrak{g},[,]_{p}, P_{\Omega}, p\right)$, where $[,]_{p}:=p \circ[$,$] , is a matching Hom-Lie Rota-Baxter algebra.$

Proof. Since $\left[p(x),[y, z]_{p}\right]_{p}=p[p(x), p[y, z]]=p^{2}[x,[y, z]]$, the Hom-Jacobi identity for $\left(\mathfrak{g},[,]_{p}, p\right)$ follows from the Jacobi identity of $(\mathfrak{g},[]$,$) . The skew-symmetry of \left(\mathfrak{g},[,]_{p}, p\right)$ holds from the skew-symmetry of $(\mathfrak{g},[]])$; hence, $\left.(\mathfrak{g},[],]_{p}, p\right)$ is a Hom-Lie algebra.

For $x, y \in \mathfrak{g}$ and $\alpha, \beta \in \Omega$, we have

$$
\begin{aligned}
{\left[P_{\alpha}(x), P_{\beta}(y)\right]_{p}=} & p\left[P_{\alpha}(x), P_{\beta}(y)\right]=p\left(P_{\alpha}\left(\left[x, P_{\beta}(y)\right]\right)\right. \\
& \left.+P_{\beta}\left[P_{\alpha}(x), y\right]+\lambda_{\beta} P_{\alpha}([x, y])\right) \\
= & P_{\alpha}\left(p\left[x, P_{\beta}(y)\right]\right)+P_{\beta}\left(p\left[P_{\alpha}(x), y\right]\right) \\
& +\lambda_{\beta} P_{\alpha}(p[x, y])=P_{\alpha}\left(\left[x, P_{\beta}(y)\right]_{p}\right) \\
& +P_{\beta}\left(\left[P_{\alpha}(x), y\right]_{p}\right)+\lambda_{\beta} P_{\alpha}\left([x, y]_{p}\right),
\end{aligned}
$$

as required.

Proposition 33. Let $\left(\mathfrak{g},[], P_{\Omega}, p\right)$ be a matching Hom-Lie Rota-Baxter algebra such that $p \circ P_{\omega}=P_{\omega} \circ p$ for each $\omega \in \Omega$. Then $\left(g,[,]_{p^{-1}}:=p^{-1} \circ[], P_{\Omega}\right)$ is a matching Lie Rota-Baxter algebra.

Proof. Since $\left[x,[y, z]_{p^{-1}}\right]_{p^{-1}}=p^{-1}\left[x, p^{-1}[y, z]\right]$, the Jacobi identity of $\left(g,[,]_{p^{-1}}\right.$ holds from the Hom-Jacobi identity of $(\mathfrak{g},[]$, , $p)$. The skew-symmetry of $\left(g,[,]_{p^{-1}}\right.$ holds from skew symmetry of $(\mathfrak{g},[], p)$; hence, $\left(g,[,]_{p^{-1}}\right.$ is a Lie algebra.

Since $p \circ P_{\omega}=P_{\omega} \circ p, p^{-1} \circ P_{\omega}=P_{\omega} \circ p^{-1}$. Then,

$$
\begin{aligned}
{\left[P_{\alpha}(x), P_{\beta}(y)\right]_{p^{-1}}=} & p^{-1}\left(\left[P_{\alpha}(x), P_{\beta}(y)\right]\right)=p^{-1}\left(P_{\alpha}\left(\left[x, P_{\beta}(y)\right]\right)\right. \\
& \left.+P_{\beta}\left(\left[P_{\alpha}(x), y\right]\right)+\lambda_{\beta} P_{\alpha}([x, y])\right) \\
= & P_{\alpha}\left(p^{-1}\left(\left[x, P_{\beta}(y)\right]\right)\right)+P_{\beta}\left(p^{-1}\left(\left[P_{\alpha}(x), y\right]\right)\right) \\
& +\lambda_{\beta} P_{\alpha}\left(p^{-1}([x, y])\right)=P_{\alpha}\left(\left[x, P_{\beta}(y)\right]_{p^{-1}}\right) \\
& +P_{\beta}\left(\left[P_{\alpha}(x), y\right]_{p^{-1}}\right)+\lambda_{\beta} P_{\alpha}\left([x, y]_{p^{-1}}\right),
\end{aligned}
$$

as required.

Definition 34. Let $(\mathfrak{g},[], p)$ be a multiplicative Hom-Lie algebra and $n \geq 0$. The $n$th derived Hom-algebra of $\mathfrak{g}$ is defined by

$$
\mathfrak{g}_{(n)}=\left(\mathfrak{g},[,]^{(n)}=p^{n} \circ[,], p^{n+1}\right) .
$$

Theorem 35. Let $\left(\mathfrak{g},[], P_{\Omega}, p\right)$ be a multiplicative matching Hom-Lie Rota-Baxter algebra and assume that $p \circ P_{\omega}=P_{\omega} \circ p$ for each $\omega \in \Omega$. Then its $n$th derived Hom-algebra is a matching Hom-Lie Rota-Baxter algebra.

Proof. Following [54], the $n$-th derived Hom-algebra is a Hom-Lie algebra. For $x, y \in \mathfrak{g}$ and $\alpha, \beta \in \Omega$,

$$
\begin{aligned}
{\left[P_{\alpha}(x), P_{\beta}(y)\right]^{(n)}=} & p^{n}\left(\left[P_{\alpha}(x), P_{\beta}(y)\right]\right)=p^{n}\left(P_{\alpha}\left(\left[x, P_{\beta}(y)\right]\right)\right. \\
& \left.+P_{\beta}\left(\left[P_{\alpha}(x), y\right]\right)+\lambda_{\beta} P_{\alpha}([x, y])\right) \\
= & P_{\alpha}\left(p^{n}\left(\left[x, P_{\beta}(y)\right]\right)\right)+P_{\beta}\left(p^{n}\left(\left[P_{\alpha}(x), y\right]\right)\right) \\
& +\lambda_{\beta} P_{\alpha}\left(p^{n}([x, y])\right)=P_{\alpha}\left(\left[x, P_{\beta}(y)\right]^{(n)}\right) \\
& +P_{\beta}\left(\left[P_{\alpha}(x), y\right]^{(n)}\right)+\lambda_{\beta} P_{\alpha}\left([x, y]^{(n)}\right)
\end{aligned}
$$

as required.

In the following, we construct matching Hom-Lie RotaBaxter algebras involving elements of the centroid of matching Lie Rota-Baxter algebras. Let $(\mathfrak{g},[], \Omega, R)$ be a matching Lie Rota-Baxter algebra. The centroid is defined by

$$
\operatorname{Cent}(\mathfrak{g}):=\{p \in \operatorname{End}(\mathfrak{g}): p[x, y]=[p(x), y], \forall x, y \in \mathfrak{g}\}
$$

Proposition 36. Let $\left(\mathfrak{g},[], P_{\Omega}\right)$ be a matching Lie Rota-Baxter algebra. Let $p \in \operatorname{Cent}(\mathfrak{g})$ and set for $x, y \in \mathfrak{g}$

$$
[x, y]_{p}^{1}:=[p(x), y] \text { and }[x, y]_{p}^{2}:=[p(x), p(y)] .
$$

Assume that $p \circ P_{\omega}=P_{\omega} \circ p$ for each $\omega \in \Omega$. Then, $\left(\mathfrak{g},[,]_{p}^{1}\right.$, $\left.P_{\Omega}, p\right)$ and $\left(\mathfrak{g},[,]_{p}^{2}, P_{\Omega}, p\right)$ are matching Hom-Lie Rota-Baxter algebras

Proof. Following Proposition 1.12 of [4], $\left.(\mathfrak{g},[]]_{p}^{1}, p\right)$ and $(\mathfrak{g}$, $\left.[,]_{p}^{2}, p\right)$ are Hom-Lie algebras. Also,

$$
\begin{aligned}
{\left[P_{\alpha}(x), P_{\beta}(y)\right]_{p}^{1}=} & {\left[p\left(P_{\alpha}(x)\right), P_{\beta}(y)\right]=p\left(\left[P_{\alpha}(x), P_{\beta}(y)\right]\right) } \\
= & p\left(P_{\alpha}\left(\left[x, P_{\beta}(y)\right]\right)+P_{\beta}\left(\left[P_{\alpha}(x), y\right]\right)\right. \\
& \left.+\lambda_{\beta} P_{\alpha}([x, y])\right)=P_{\alpha}\left(\left[p(x), P_{\beta}(y)\right]\right) \\
& +P_{\beta}\left(\left[p\left(P_{\alpha}(x)\right), y\right]\right)+\lambda_{\beta} P_{\alpha}([p(x), y]) \\
= & P_{\alpha}\left(\left[x, P_{\beta}(y)\right]_{p}^{1}\right)+P_{\beta}\left(\left[P_{\alpha}(x), y\right]_{p}^{1}\right) \\
& +\lambda_{\beta} P_{\alpha}\left([x, y]_{p}^{1}\right)
\end{aligned}
$$


and

$$
\begin{aligned}
{\left[P_{\alpha}(x), P_{\beta}(y)\right]_{p}^{2}=} & {\left[p\left(P_{\alpha}(x)\right), p\left(P_{\beta}(y)\right)\right]=p\left(\left[P_{\alpha}(x), p\left(P_{\beta}(y)\right)\right]\right) } \\
= & -p^{2}\left(\left[P_{\beta}(y), P_{\alpha}(x)\right]\right)=p^{2}\left(\left[P_{\alpha}(x), P_{\beta}(y)\right]\right) \\
= & p^{2}\left(P_{\alpha}\left(\left[x, P_{\beta}(y)\right]\right)+P_{\beta}\left(\left[P_{\alpha}(x), y\right]\right)\right. \\
& \left.+\lambda_{\beta} P_{\alpha}([x, y])\right)=P_{\alpha}\left(\left[p(x), p\left(P_{\beta}(y)\right)\right]\right) \\
& +P_{\beta}\left(\left[p\left(P_{\alpha}(x)\right), p(y)\right]\right)+\lambda_{\beta} P_{\alpha}([p(x), p(y)]) \\
= & P_{\alpha}\left(\left[x, P_{\beta}(y)\right]_{p}^{2}\right)+P_{\beta}\left(\left[P_{\alpha}(x), y\right]_{p}^{2}\right) \\
& +\lambda_{\beta} P_{\alpha}\left([x, y]_{p}^{2}\right) .
\end{aligned}
$$

This completes the proof.

Proposition 37. Let $\left(A,[],, P_{\Omega}, p\right)$ be a matching Hom-Lie Rota-Baxter algebra of weight zero (i.e. $\lambda_{\omega}=0$ for all $\omega \in \Omega$ ). Assume that $p \circ P_{\omega}=P_{\omega} \circ p$ for each $\omega \in \Omega$. Then, $\left(A,\left\{*_{\omega}\right.\right.$ $\mid \omega \in \Omega\}, p)$ is a matching Hom-pre-Lie algebra, where

$$
x *{ }_{\omega} y=\left[P_{\omega}(x), y\right] \text { for } x, y \in A \text { and } \omega \in \Omega .
$$

Proof. For $x, y, z \in \mathfrak{g}$ and $\alpha, \beta \in \Omega$, we have

$$
\begin{aligned}
& p(x) *_{\alpha}\left(y *_{\beta} z\right)-\left(x *_{\alpha} y\right) *_{\beta} z \\
&=\left[P_{\alpha}(p(x)),\left[P_{\beta}(y), z\right]\right]-\left[P_{\beta}\left(\left[P_{\alpha}(x), y\right]\right), p(z)\right] \\
&(\text { by Eq. }(91)) \\
&= {\left[P_{\alpha}(p(x)),\left[P_{\beta}(y), z\right]\right]-\left[\left[P_{\alpha}(x), P_{\beta}(y)\right], p(z)\right] } \\
&+\left[P_{\alpha}\left(\left[x, P_{\beta}(y)\right]\right), p(z)\right](\text { by Eq. }(82)) \\
&= {\left[p\left(P_{\alpha}(x)\right),\left[P_{\beta}(y), z\right]\right]+\left[p(z),\left[P_{\alpha}(x), P_{\beta}(y)\right]\right] } \\
&-\left[P_{\alpha}\left(\left[P_{\beta}(y), x\right]\right), p(z)\right]\left(\text { by p o } \mathrm{P}_{\alpha}=\mathrm{P}_{\alpha} \circ \mathrm{p}\right) \\
&=-\left[p\left(P_{\beta}(y)\right),\left[z, P_{\alpha}(x)\right]\right]-\left[P_{\alpha}\left(\left[P_{\beta}(y), x\right]\right), p(z)\right] \\
&(\text { by Hom }-\operatorname{Jacobi~identity)} \\
&= {\left[P_{\beta}(p(y)),\left[P_{\alpha}(x), z\right]\right]-\left[P_{\alpha}\left(\left[P_{\beta}(y), x\right]\right), p(z)\right] } \\
&= p(y) *{ }_{\beta}\left(x *_{\alpha} z\right)-\left(y *{ }_{\beta} x\right) *{ }_{\alpha} p(z) .
\end{aligned}
$$

This completes the proof.

\section{Data Availability}

No data were used to support this study.

\section{Disclosure}

No potential conflict of interest was reported by the authors.

\section{Conflicts of Interest}

The authors declare that they have no conflicts of interest.

\section{Acknowledgments}

The authors would like to thank the anonymous reviewers for their valuable comments which improved the paper. This work was supported by the National Natural Science Foundation of China (grant no. 11771191) and the Fundamental Research Funds for the Central Universities (grant no. lzujbky-2020-12). The work of Chia Zargeh was partially supported by CNPq grant.

\section{References}

[1] J. T. Hartwig, D. Larsson, and S. D. Silvestrov, "Deformations of Lie algebras using $\sigma$-derivations," Journal of Algebra, vol. 295, pp. 3140-3361, 2006.

[2] Y. Chen, Z. Wang, and L. Zhang, "Quasitriangular Hom-Lie bialgebras," Journal of Lie Theory, vol. 22, pp. 1075-1089, 2012.

[3] Y. Chen, H. Zheng, and L. Zhang, "Double Hom-Associative Algebra and Double Hom-Lie Bialgebra," Advances in Applied Clifford Algebras, vol. 30, article 8, 2020.

[4] S. Benayadi and A. Makhlouf, "Hom-Lie algebras with symmetric invariant nondegenerate bilinear forms," Journal of Geometry and Physics, vol. 76, pp. 38-60, 2014.

[5] S. Zheng and L. Guo, "Free involutive Hom-semigroups and Hom-associative algebras," Frontiers of Mathematics in China, vol. 11, no. 2, pp. 497-508, 2016.

[6] D. Larsson and S. D. Silvestrov, "Quasi-hom-lie algebras, central extensions and 2-cocycle-like identities," Journal of Algebra, vol. 288, no. 2, pp. 321-344, 2005.

[7] Y. Sheng, "Representations of hom-lie algebras," Algebras and Representation Theory, vol. 15, no. 6, pp. 1081-1098, 2012.

[8] J. Zhao, L. Chen, and L. Ma, "Representations andT*-Extensions of hom-Jordan-Lie algebras," Communications in Algebra, vol. 44, no. 7, pp. 2786-2812, 2016.

[9] F. Ammar, Z. Ejbehi, and A. Makhlouf, "Cohomology and deformations of Hom-algebras," Journal of Lie Theory, vol. 21, pp. 813-836, 2011.

[10] D. Yau, "Hom-algebras and homology," Journal of Lie Theory, vol. 19, pp. 409-421, 2009.

[11] S. Wang and S. Wang, "Hom-lie algebras in Yetter-Drinfeld categories," Communications in Algebra, vol. 42, no. 10, pp. 4526-4547, 2014.

[12] Y. Chen and L. Zhang, "Hom-Operators and Hom-YangBaxter Equations," Advances in Mathematical Physics, vol. 2015, Article ID 823756, 11 pages, 2015.

[13] Y. Chen and L. Zhang, "Hom-Yang-Baxter equations and Frobenius monoidal Hom-algebras," Advances in Mathematical Physics, vol. 2018, Article ID 2912578, 2018.

[14] L. Liu, A. Makhlouf, C. Menini, and F. Panaite, “ $(\sigma, \tau)$-RotaBaxter operators, infinitesimal Hom-bialgebras and the associative (Bi)Hom-Yang-Baxter equation," Canadian Mathematical Bulletin, vol. 62, pp. 355-372, 2019.

[15] Y. Sheng and C. Bai, "A new approach to hom-lie bialgebras," Journal of Algebra, vol. 399, pp. 232-250, 2014.

[16] D. Yau, "The Hom-Yang-Baxter equation and Hom-lie algebras," Journal of Mathematical Physics, vol. 52, no. 5, article 053502, 2011.

[17] Y. Sheng and D. Chen, "Hom-Lie 2-algebras," Journal of Algebra, vol. 376, pp. 174-195, 2013. 
[18] L. Song and R. Tang, "Derivation Hom-lie 2-algebras and nonabelian extensions of regular Hom-lie algebras," Journal of Algebra and Its Applications, vol. 17, no. 5, article 1850081, 2018.

[19] T. Ma and H. Zheng, “( $m, n)$-Hom-Lie algebras," Publicationes Mathematicae Debrecen, vol. 92, pp. 59-78, 2018.

[20] A. Makhlouf and S. Silvestrov, "Hom-algebra structures," Journal of Generalized Lie Theory and Applications, vol. 2, no. 2, pp. 51-64, 2008.

[21] L. Guo, B. Zhang, and S. Zheng, "Universal enveloping algebras and Poincaré-Birkhoff-Witt theorem for involutive Hom-lie algebras," Journal of Lie Theory, vol. 26, pp. 739$759,2018$.

[22] Q. Jin and X. Li, "Hom-Lie algebra structures on semi-simple Lie algebras," Journal of Algebra, vol. 319, no. 4, pp. 13981408, 2008.

[23] A. Makhlouf and P. Zusmanovich, "Hom-lie structures on Kac-moody algebras," Journal of Algebra, vol. 515, pp. 278297, 2018.

[24] A. Makhlouf and S. Silvestrov, "Hom-Lie Admissible HomCoalgebras and Hom-Hopf Algebras," in Generalized Lie Theory in Mathematics, Physics and Beyond, S. Silvestrov, E. Paal, V. Abramov, and A. Stolin, Eds., pp. 189-206, Springer, Berlin, 2009.

[25] A. Makhlouf and S. Silvestrov, "Hom-algebras and Hom-coalgebras," Journal of Algebra and Its Applications, vol. 9, pp. 553-589, 2011.

[26] M. Hassanzadeh, "Hom-groups, representations and homological algebra," Colloquium Mathematicum, vol. 158, no. 1, pp. 21-38, 2019.

[27] M. Hassanzadeh, "Lagrange's theorem for Hom-groups," The Rocky Mountain Journal of Mathematics, vol. 49, no. 3, pp. 773-787, 2019.

[28] S. Guo, X. Zhang, and S. Wang, "Relative Hom-Hopf modules and total integrals," Journal of Mathematical Physics, vol. 56, no. 2, article 021701, 2015.

[29] H. Albuquerque, E. Barreiro, A. J. Calderón, and J. M. Sánchez, "On split regular Hom-Lie superalgebras," Journal of Geometry and Physics, vol. 128, pp. 1-11, 2018.

[30] L. Ma, L. Chen, and J. Zhao, " $\delta$-hom-Jordan Lie superalgebras," Communications in Algebra, vol. 46, pp. 1668-1697, 2017.

[31] T. Ma, L. Dong, and H. Li, "General Hom-Lie algebra," Journal of Algebra and Its Applications, vol. 15, no. 5, article 1650081, 2016.

[32] M. J. Argón Periñán and A. J. Calderó Martín, “The structure of split regular Hom-Poisson algebras," Colloquium Mathematicum, vol. 145, pp. 1-13, 2016.

[33] J. L. Loday, "DialgebrasDialgebras and Related Operads," vol. 1763 of Lecture Notes in Math, Springer, Berlin, Heidelberg, 2001.

[34] J. L. Loday and M. Ronco, "Cofree Hopf algebras," Comptes Rendus Mathematique, vol. 337, no. 3, pp. 153-158, 2003.

[35] M. Aguiar, "Pre-Poison algebras," Letters in Mathematical Physics, vol. 54, pp. 263-277, 2000.

[36] K. Ebrahimi-Fard, "Loday-type algebras and the Rota-Baxter relation," Letters in Mathematical Physica, vol. 61, pp. 130147, 2002.

[37] A. Makhlouf, "Hom-dendriform algebras and Rota-Baxter Hom-algebras," in Operads and Universal Algebra, pp. 147171, Hackensack, NJ, 2012.
[38] Y. Zhang, X. Gao, and L. Guo, "Matching Rota-Baxter algebras, matching dendriform algebras and matching pre- Lie algebras," Journal of Algebra, vol. 552, pp. 134-170, 2020.

[39] L. Foissy, "Algebraic structures on typed decorated rooted trees," https://arxiv.org/abs/1811.07572.

[40] Y. Bruned, M. Hairer, and L. Zambotti, "Algebraic renormalisation of regularity structures," Inventiones Mathematicae, vol. 215, no. 3, pp. 1039-1156, 2019.

[41] M. Aguiar, "On the associative analog of lie bialgebras," Journal of Algebra, vol. 54, pp. 263-277, 2000.

[42] X. Gao, L. Guo, and Y. Zhang, Commutative Matching RotaBaxter Operators, Decorated Shuffle Products and Matching Zinbiel Algebras, 2020.

[43] J. Liu, C. Bai, and Y. Sheng, "Compatible $\mathcal{O}$-operators on bimodules over associative algebras," Journal of Algebra, vol. 532, pp. 80-118, 2019.

[44] Y. Zhang, C. Bai, and L. Guo, "The category and Operad of matching dialgebras," Applied Categorical Structures, vol. 21, no. 6, pp. 851-865, 2013.

[45] Y. Zhang, C. Bai, and L. Guo, "Totally compatible associative and lie dialgebras, tridendriform algebras and PostLie algebras," Science China Mathematics, vol. 57, no. 2, pp. 259-273, 2014.

[46] Y. Y. Zhang and X. Gao, "Free Rota-Baxter family algebras and (tri)dendriform family algebras," Pacific Journal of Mathematics, vol. 301, no. 2, pp. 741-766, 2019.

[47] Y. Y. Zhang, X. Gao, and D. Manchon, "Free (tri)dendriform family algebras," Journal of Algebra, vol. 547, pp. 456-493, 2020.

[48] D. Chen, Y. F. Luo, Y. Zhang, and Y. Y. Zhang, Free $\Omega$-RotaBaxter Algebras and Gröbner-Shirshov Bases, Accepted by International Journal of Algebra and Computation, International journal of Algebra and Computation, 2020.

[49] X. Gao, L. Guo, and Y. Zhang, "Hopf algebra of multidecorated rooted forests, free matching Rota-Baxter algebras and Gröbner-Shirshov bases," https://arxiv.org/abs/2002 .02864 .

[50] L. Guo, R. Gustavson, and Y. Li, "An algebraic study of Volterra integral equations and their operator linearity," 2020, This paper is just submitted now.

[51] M. A. Semenov-Tian-Shansky, "What is a classical $r$-matrix?," Functional Analysis and Its Applications, vol. 17, pp. 259-272, 1983.

[52] D. Yau, "Hom-algebras as deformations and homology," Journal of Lie Theory, vol. 19, pp. 409-421, 2009.

[53] A. Gohr, "On Hom-algebras with surjective twisting," Journal of Algebra, vol. 324, no. 7, pp. 1483-1491, 2010.

[54] D. Yau, "Hom-Malsev, Hom-alternative, and Hom-Jordan algebras," https://arxiv.org/abs/1002.3944.

[55] A. A. Belavin and V. G. Drinfeld, "Solutions of the classical Yang-Baxter equation for simple lie algebras," Functional Analysis and its Applications, vol. 16, pp. 159-180, 1982. 